\title{
Characterization of the Transthyretin Acid Denaturation Pathways by Analytical Ultracentrifugation: Implications for Wild-Type, V30M, and L55P Amyloid Fibril Formation $^{\dagger}$
}

\author{
Hilal A. Lashuel, $\stackrel{\ddagger}{ }$ Zhihong Lai, $\stackrel{\ddagger}{ }$ and Jeffery W. Kelly* \\ Department of Chemistry and The Skaggs Institute of Chemical Biology, The Scripps Research Institute, \\ 10550 North Torrey Pines Road, MB12, La Jolla, California 92037
}

Received August 5, 1998

\begin{abstract}
Analytical ultracentrifugation methods were utilized to further characterize the acid denaturation pathways of wild-type, V30M, and L55P transthyretin (TTR) that generate intermediates leading to amyloid fibril formation and possibly the diseases senile systemic amyloidosis and familial amyloid polyneuropathy. Equilibrium and velocity methods were employed herein to characterize the TTR quaternary structural requirements for amyloid fibril formation. From neutral to slightly acidic conditions ( $\mathrm{pH} 7.5-5.1)$, wildtype transthyretin $\left(0.2-0.3 \mathrm{mg} / \mathrm{mL}, 100 \mathrm{mM} \mathrm{KCl}, 37^{\circ} \mathrm{C}\right)$ exists as a tetramer and is incapable of fibril formation. Under more acidic conditions ( $\mathrm{pH} 5$ to 3.9), tetrameric wild-type TTR slowly dissociates to a monomer having an alternatively folded tertiary structure(s) that self-assembles at physiological concentration $(0.2 \mathrm{mg} / \mathrm{mL})$ into a ladder of quaternary structural intermediates of increasing molecular weight. These intermediates appear to be on the pathway of amyloid fibril formation, since they ultimately disappear when amyloid fibrils are observed. The V30M and L55P TTR variants exhibit similar acid denaturation pathways, with the exception that dissociation of the tetramer to the monomeric amyloidogenic intermediate occurs at a higher $\mathrm{pH}$ and to a much greater extent, allowing the quaternary structural intermediates to be readily observed by velocity methods. Partial denaturation and assembly of the monomeric amyloidogenic intermediate(s) occur at $\mathrm{pH} 5.4$ for V30M and L55P TTR over a $72 \mathrm{~h}$ period, during which wild-type TTR maintains its normal tetrameric three-dimensional structure. Interestingly, the L55P and V30M familial amyloid polyneuropathy (FAP) associated variants form amyloid protofilaments at $\mathrm{pH} 7.5\left(37^{\circ} \mathrm{C}\right)$ after several weeks of incubation, suggesting that the activation barriers for TTR tetramer dissociation to the monomeric amyloidogenic intermediate are much lower for the FAP variants relative to wild-type TTR, which does not form amyloid or amyloid protofilaments under these conditions. This study establishes the key role of the monomeric amyloidogenic intermediate and its selfassembly into a ladder of quaternary structural intermediates for the formation of wild-type, V30M, and L55P transthyretin amyloid fibrils.
\end{abstract}

Amyoid fibril formation refers to an abnormal and likely pathogenic process whereby a human amyloidogenic protein undergoes a conformational change and self-assembles into fibrils having a characteristic cross $\beta$-structure $(1-12)$. Although the fibrils associated with different amyloid diseases (neurodegenerative diseases) are made of different proteins, the amyloid fibrils share strikingly similar X-ray diffraction patterns, suggesting a related structure and a common mechanism of fibril formation $(13-15)$. Even though there is considerable evidence for a causal link between fibril formation and amyloid diseases, the exact pathological mechanism remains unclear. Until recently, amyloid fibril formation was thought to follow a two-state mechanism analogous to a crystallization process, where only the amyloidogenic conformational intermediate and amyloid fibrils are significantly populated during fibril formation.

$\doteqdot$ The authors gratefully acknowledge primary financial support of the National Institute of Health (Grant R01 DK46335-01) and secondary support from The Skaggs Institute of Chemical Biology and the Lita Annenberg Hazen Foundation.

$\doteqdot$ These authors contributed equally to this work.
However, several laboratories including our own have provided evidence supporting the existence of numerous preamyloid quaternary structural intermediates $(7,16,17)$. These intermediates seem to play a major role in the amyloid fibril formation process and possibly in the pathological mechanism of amyloid disease $(2,17,18)$.

Transthyretin (TTR) ${ }^{1}$ is one of twenty human amyloidogenic proteins that change conformation and abnormally self-assemble into fibrilar quaternary structures under partially denaturing conditions $(1,6,8,9,19,20)$. Transthyretin is known to be the major component of the amyloid deposits that putatively cause the human amyloid diseases senile systemic amyloidosis (SSA) and familial amyloid polyneuropathy (FAP) $(5,21)$. Wild-type TTR composes the fibrils in the senile form of the disease, whereas 1 of 54 single site variants predominantly constitute the fibrils in the familial

\footnotetext{
${ }^{1}$ Abbreviations: TTR, transthyretin; FAP, familial amyloid polyneuropathy; SSA, senile systemic amyloidosis; SDS-PAGE, sodium dodecyl sulfate-polyacrylamide gel electrophoresis; CD, circular dichroism; $s$, sedimentation coefficient; S, Svedberg unit of $\left(10^{-13} \mathrm{~s}\right)$; $D$, diffusion coefficient; EM, electron microscopy.
} 
disease (22). Among these, V30M is the most frequent FAPassociated variant and is of intermediate stability, while L55P is the most pathogenic and unstable FAP variant characterized to date $(5,22-25)$. Thus the amyloidogenic mutations appear to alter the acid denaturation pathway by making either the thermodynamics or the kinetics, or both, favorable for the formation of the amyloidogenic intermediate that selfassembles into amyloid fibrils $(1-3,7,23,24,26-32)$. The $130 \AA$ diameter transthyretin amyloid fibril is made up of four protofilaments, each having a characteristic cross- $\beta$ repeat structure. The individual $\beta$-strands composing the fibril are oriented perpendicular to the long axis of the protofilaments composing the fibril (33). Recent synchrotron X-ray fibril diffraction data combined with high-resolution electron microscopy studies on V30M TTR amyloid suggest a structural model where the constituent cross $\beta$-strands in the protofilaments are twisted $15^{\circ}$ with respect to each other, forming a complete $\beta$-helical turn every $24 \beta$-strands (34).

Normally folded wild-type, V30M, and L55P TTR can be converted into amyloid fibrils either by partial acid denaturation or during $\mathrm{pH}$-mediated reconstitution (refolding), implying that an intermediate on the denaturation/ reconstitution pathway is the amyloidogenic intermediate that self-assembles into amyloid fibrils $(23,24,26,29)$. Earlier data from our laboratory show that the V30M and L55P variants can form amyloid fibrils under mildly acidic conditions (e.g. pH 5.4), where wild-type TTR is a nonamyloidogenic conformationally stable tetramer $(1-3,7,23$, $24,26,29)$. Recent data described herein demonstrate that the V30M and L55P FAP-associated variants are capable of forming amyloid protofilaments at physiological $\mathrm{pH}$ and concentration upon extended incubation (weeks) at $37{ }^{\circ} \mathrm{C}$. Electron microscopy was employed to observe the protofilaments because light scattering is not sensitive enough to detect these protofilaments. Importantly, the wild-type protein is incapable of fibril or protofilament formation under these conditions, even after months of incubation. These results suggest that wild-type TTR cannot dissociate to monomer under mild conditions, unlike the FAP variants which have lower partial denaturation barriers that facilitate the formation of the monomeric amyloidogenic intermediate $(23,24,30$, 31). Amyloid fibrils prepared by partial acid denaturation or reconstitution are very similar to fibrils formed in vivo on the basis of X-ray diffraction, EM morphology, and dye binding properties. This structural similarity confirms that the amyloid fibrils form as a result of specific proteinprotein interactions and not a result of another process such as isoelectric precipitation, which needs to be considered here since the $\mathrm{pH}$ of maximal TTR fibril formation $(\mathrm{pH} 4.4)$ is near the PI of TTR (4.6-4.95).

Quintas and co-workers have evaluated the quaternary structural changes exhibited by wild-type, V30M, L55P, and T119M TTR in neutral $\mathrm{pH}$ solution by gel filtration chromatography, where dissociation is mediated by dilution of TTR solutions into the sub-micromolar concentration range. They succeeded in identifying the presence of soluble oligomeric species at $\mathrm{pH} 7$ for V30M and L55P TTR; however, owing to the limitations of gel filtration, we cannot learn very much about the size and distribution of the oligomeric species (35). Evaluation of the self-assembly pathway(s) leading to amyloid formation has been hindered by the available methods for identifying quaternary structural intermediates. Speed et al. utilized nondenaturing gel electrophoresis to study the aggregation of the P22 tail spike protein successfully (36). Unfortunately, dissection of transthyretin fibril formation is not amenable to this approach, as quaternary structural intermediates are not observed as a ladder of bands; instead streaking on the PAGE gels is observed. Holzman and co-workers utilized sedimentation velocity studies and Teplow and Murphy used light scattering $(37-40)$ to study the assembly of the $\mathrm{A} \beta$-protein into amyloid, both methods identifying the presence of oligomeric intermediates. More recently, Lansbury and Krafft have utilized atomic force microscopy to examine the time course of $\mathrm{A} \beta$ amyloid fibril formation, identifying a series of intermediates preceding the formation of amyloid fibrils (16, 18). Advances in analytical ultracentrifugation methods make the observation and characterization of quaternary structural intermediates quite straightforward (41-43).

Herein, sedimentation velocity and equilibrium ultracentrifugation methods are used to carefully characterize the TTR quaternary structural changes as a function of $\mathrm{pH}$ to identify the quaternary structural requirements for amyloid fibril formation and to identify possible intermediates involved in amyloid fibril formation. These studies show that TTR $(0.2-0.3 \mathrm{mg} / \mathrm{mL}$ ) under acidic conditions (e.g. pH 4.4) dissociates to a monomeric intermediate to varying degrees, depending on the TTR sequence under study, and selfassembles into an array of quaternary structure intermediates differing in MW by approximately one monomer unit. The self-assembly of these intermediate quaternary structures leads to significant amyloid fibril formation at elevated temperature $\left(37^{\circ} \mathrm{C}\right)$, by a pathway that is currently under further scrutiny employing electron microscopy, atomic force microscopy, and related approaches. At low pH (3-2) in the presence of salt, TTR is also capable of assembly into low-MW quaternary structures; however, these assemblies do not yield amyloid fibrils.

\section{MATERIALS AND METHODS}

Transthyretin was purified from an E. coli expression system described previously (23). Purification involves liberating TTR and other proteins from the periplasmic space of $E$. coli by osmotic shock. The resulting solution is concentrated 5 -fold. The protein pellet produced by a 70$85 \%$ ammonium sulfate precipitation is then dissolved and subjected to DEAE ion exchange chromatography, resulting in TTR with $>95 \%$ purity. All reagents used were of the highest purity available from Sigma or USB.

Probing the pH-Dependent Quaternary Structure Changes by Sedimentation Velocity. Concentrated TTR stock solutions $(5 \mathrm{mg} / \mathrm{mL})$ were spun down on a desktop centrifuge for 15 min at $4{ }^{\circ} \mathrm{C}$, and all buffers were filtered through $0.22 \mu \mathrm{m}$ filters before use in analytical ultracentrifugation experiments. TTR solutions at the indicated concentration $(0.2$ or 0.02 $\mathrm{mg} / \mathrm{mL})$ and the desired $\mathrm{pH}(50 \mathrm{mM}$ phosphate or acetate buffer in $100 \mathrm{mM} \mathrm{KCl}$ ) were prepared by dilution or by dialysis against the desired buffer, incubated at $4{ }^{\circ} \mathrm{C}$ for at least $12 \mathrm{~h}$, and brought up to $25^{\circ} \mathrm{C}$ for several hours prior to the run (except in cases specified as being analyzed at 4 ${ }^{\circ} \mathrm{C}$ ). The sedimentation properties of wild-type, V30M, and L55P TTR incubated for $24 \mathrm{~h}$ at $\mathrm{pHs} 8.0,7.0,6.0,5.5,5.0$, $4.4,4.0,3.0$, and 2.0 were obtained from data collected on 
a temperature controlled Beckman XL-A or XL-I analytical ultracentrifuge equipped with a An60Ti rotor and photoelectric scanner. A double sector cell, equipped with a 12 $\mathrm{mm}$ Epon centerpiece and quartz windows, was loaded with $400-420 \mathrm{~mL}$ of sample using a blunt-end microsyringe. Data were collected at rotor speeds of 3000-60000 rpm in continuous mode at $25^{\circ} \mathrm{C}$ ( or $4{ }^{\circ} \mathrm{C}$ ), with a step size of 0.005 $\mathrm{cm}$ and an average of four-scans per point. Fewer scans per point were used when fast sedimenting species were followed to allow more data sets per unit time to be collected.

Analysis of the Sedimentation Velocity Profile of TTR. The second moment boundary sedimentation analysis was applied to determine the sedimentation coefficients $(s)$, which are reported in Svedberg units $\left(\mathrm{S}, 10^{-13} \mathrm{~s}\right)$ for a given quaternary structure under conditions where a single ideal species exists in solution, as discerned previously by sedimentation equilibrium. For boundary sedimentation analysis, the movement of the midpoint of the absorbance boundary $(r)$ vs time $(t)$ was used to obtain the uncorrected sedimentation coefficient, $\mathrm{s}$, from the slope of the plot of $\ln (r)$ vs $t / \omega^{2}$; i.e., $\left[\mathrm{d} \ln (r) / \omega^{2}\right.$ $\mathrm{d} t]$. The observed sedimentation coefficient, $s$, was corrected to standard conditions (water at $20^{\circ} \mathrm{C}$ ) with the following equation using tabulated density and viscosity data:

$$
s_{20, \mathrm{w}}=s \frac{(\eta)_{\mathrm{T}, \mathrm{b}}}{(\eta)_{20, \mathrm{w}}} \cdot \frac{(1-\bar{v} \rho)_{20, \mathrm{w}}}{(1-\bar{v} \rho)_{\mathrm{T}, \mathrm{b}}}
$$

where $\rho$ and $\eta$ are the density and viscosity, respectively, of water at $20{ }^{\circ} \mathrm{C}$, and $\bar{v}$ is the partial specific volume of the protein. The density and viscosity were calculated using polynomial equations and tables of coefficients (44). The partial specific volume of TTR $\left(0.734 \mathrm{~cm}^{3} / \mathrm{g}\right)$ was estimated on the basis of the partial specific volumes of the component amino acid residues (45-47).

For heterogeneous samples containing up to three sedimenting species, the data were fit using a direct fitting analysis of the collected absorbance data. This method works well, especially for proteins with molecular weights of $10000-40000$ for which the boundaries are quite broad due to diffusion. Since the molecular weight of monomeric TTR is only 14000 , very broad boundaries dominated by diffusion are observed for the monomer and are difficult to analyze by the time derivative analysis. At this molecular weight, it is nearly impossible to get the boundary to clear the meniscus; hence, direct fitting analysis is used. A direct boundary fitting approach was applied using the Svedberg program developed by Philo, where multiple raw data sets (concentration vs radius) taken at various times during the run are simultaneously fit to approximate solutions of the Lamm equation, with $s, D$ (diffusion coefficient, with a unit of Fick, $1 \mathrm{~F}=10^{7} \mathrm{~cm}^{2} / \mathrm{s}$ ), and the loading concentration as fitting variables (48). This method has the advantage of allowing for the calculation of the molecular weight of the sedimenting species using eq II since $s$ and $D$ can be obtained from the fitting analysis.

$$
\mathrm{MW}=\frac{s R T}{D(1-\bar{v} \rho)}
$$

In the cases where the diffusion coefficient cannot be determined accurately, the molecular weight of a spherical protein can still be estimated by combining the Svedberg equation and Stokes equation for the frictional coefficient to obtain eq III, which utilizes the estimated sedimentation coefficient.

$$
(\mathrm{MW})^{2 / 3}=100 s \bar{v}^{1 / 3} /(1-\bar{v} \rho)
$$

For complicated systems containing multiple species we used the van Holde and Weischet global boundary analysis for fitting the absorbance data (42). The van Holde and Weischet global fitting method is very useful for examining sample homogeneity/heterogeneity and facilitates a rigorous analysis of complex boundaries of multicomponent systems, yielding accurate $S_{20, \mathrm{w}}$ for all components present (43). The van Holde and Weischet analysis of the data was carried out using the Ultrascan software developed by Demeler (for information on Ultrascan, http://bioc02.uthscsa.edu/.biochem/ tutor.html). The contribution of diffusion to the boundary shape is removed in the van Holde and Weischet analysis by extrapolating to infinite time $\left(t^{-1 / 2}=0\right)$, thus achieving resolution between species of similar $s$ values. The $s$ values obtained are visualized either as an extrapolation plot (e.g. Figure 1B) or as an integral distribution plot showing the distribution of $s$ values along the boundary known as $G(s)$ (e.g. Figure 1C), where the $G(s)$ plots simply represent another way of presenting the extrapolation plots. Usually we display the extrapolation plots within because of their ability to reveal details or complexities in the boundary shape that are not usually obvious by inspection of the velocity data. In the extrapolation plots a homogeneous sample with respect to both molecular weight and the frictional coefficient would yield a single $Y$ intercept corresponding to the diffusion corrected sedimentation coefficient of the species (e.g. Figure 1B), while a sample that is heterogeneous would give multiple $Y$ intercepts (e.g. Figure 6A). The van Holde and Weischet analysis is not perturbed by experimentally noisy data. Demeler and co-workers have shown that this method faithfully interprets the experimental data even at higher noise levels than are usually experienced with the XL-A (using simulated data) (49). The $\mathrm{d} c / \mathrm{d} t$ time derivative analysis method for calculating the apparent sedimentation coefficient distribution $g\left(s^{*}\right)$ developed by Stafford was also used to confirm the homogeneity/heterogeneity of the samples (data not shown) $(41,50)$.

Sedimentation Equilibrium Experiments To Determine the Molecular Weight of TTR under Acidic Denaturing Conditions in the Absence of Salt. Sedimentation equilibrium runs were performed on a TTR sample $(110 \mathrm{~mL})$ at $\mathrm{pH} 2.0$ in the absence of salt from 3000-17 $000 \mathrm{rpm}$ using a double sector cell with charcoal-filled Epon centerpieces and sapphire windows. All scans were performed at $280 \mathrm{~nm}$, with a step size of $0.001 \mathrm{~cm}$ and 50 averaged scans. Samples were allowed to equilibrate for $24 \mathrm{~h}$, and duplicate scans $3 \mathrm{~h}$ apart were overlaid to determine that equilibrium had been reached. The data were analyzed by a nonlinear least-squares analysis using the Origin software provided by Beckman. The data were then fit to a single ideal species model using the following equation to determine the best fitting molecular weight:

$$
\begin{aligned}
& A_{\mathrm{r}}= \\
& \quad \exp \left[\ln \left(A_{0}\right)+\left(M \omega^{2}(1-\bar{v} \rho) / 2 R T\right)\left(x^{2}-x_{0}{ }^{2}\right)\right]+E(\mathrm{IV})
\end{aligned}
$$



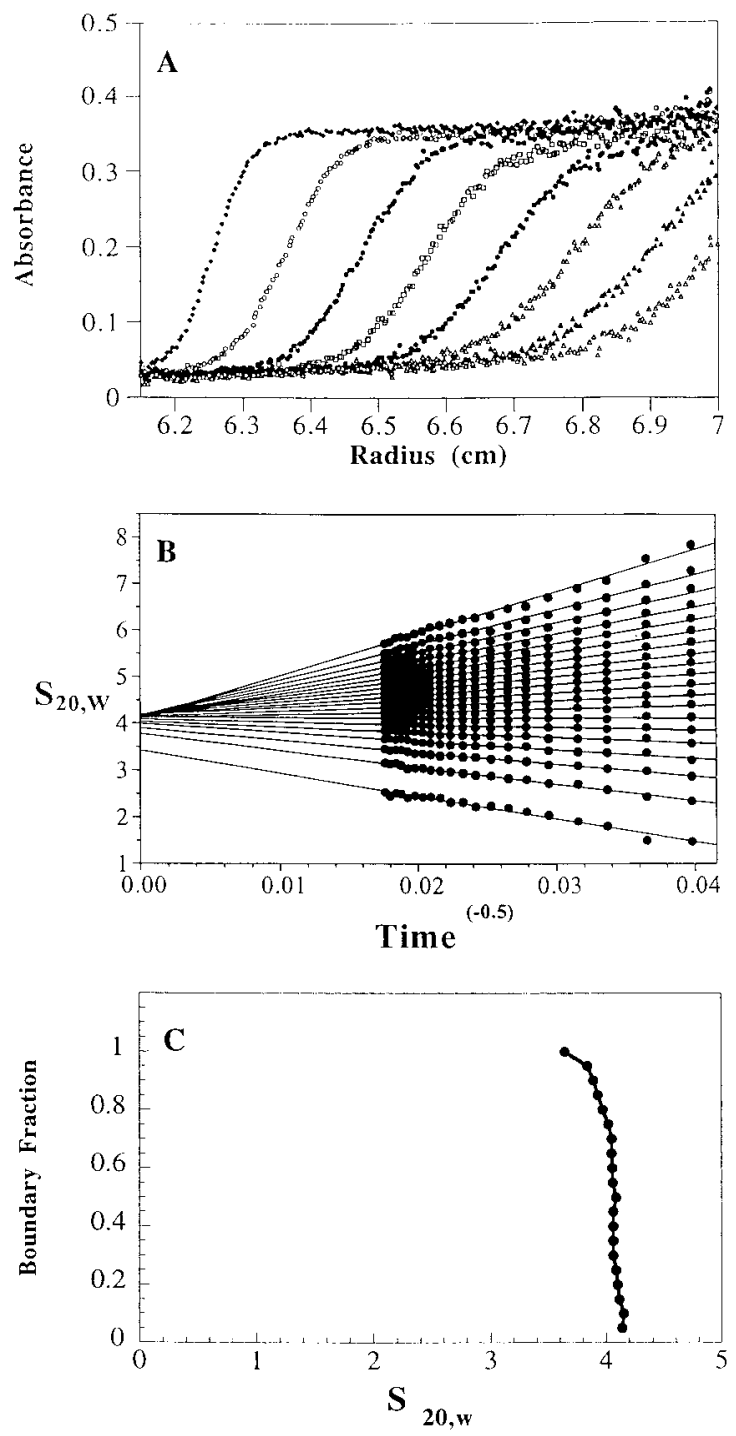

FIGURE 1: Sedimentation velocity profiles of wild-type TTR $(0.2$ $\mathrm{mg} / \mathrm{mL}$ ) at $\mathrm{pH}$ 7.0. (A) Overlay of data sets recorded approximately 15 min apart. (B) van Holde and Weischet extrapolation plots showing the tetrameric quaternary structure in solution. (C) $G(s)$ distribution plot demonstrating the tetrameric structure.

where $A_{\mathrm{r}}$ is the absorbance at radius $x, A_{0}$ is the absorbance at a reference radius $x_{0}$ (usually the meniscus), $\bar{v}$ is the partial specific volume of TTR $(\mathrm{mL} / \mathrm{g}), \rho$ is the density of the solvent $(\mathrm{g} / \mathrm{mL}), \omega$ is the angular velocity of the rotor $(\mathrm{rad} /$ s), $E$ is the base-line error correction factor, $M$ is the molecular weight, $R$ is the universal gas constant $(8.314 \times$ $\left.10^{7} \mathrm{ergs} / \mathrm{mol}\right)$, and $T$ is temperature $(\mathrm{K})$. The goodness of the fit was evaluated on the basis of the randomness and magnitude of the residuals, expressed as the difference between the theoretical curve and the experimental data, and by checking the fit parameters for physical reasonability. Transforming the equilibrium concentration gradient (absorbance vs radius) into a $M_{\mathrm{w} \text {,app }}$ vs concentration plot is also used to provide information regarding the degree of association in the system.

Far-UV Circular Dichroism of TTR at $0.02 \mathrm{mg} / \mathrm{mL}$. Transthyretin samples $(0.02 \mathrm{mg} / \mathrm{mL}, \mathrm{pH} 7,4.4$, and 3.9) were incubated at $4{ }^{\circ} \mathrm{C}$ for $12 \mathrm{~h}$ before the temperature was increased to $25^{\circ} \mathrm{C}$ for $2-3 \mathrm{~h}$. The spectra were recorded on an Aviv model 62DS spectrometer at $25{ }^{\circ} \mathrm{C}$ using a bandwidth of $1 \mathrm{~nm}$, a time constant of $4 \mathrm{~s}$, and a step size of
$0.5 \mathrm{~nm}$. A $5 \mathrm{~mm}$ quartz cell was used to collect the spectra from 250 to $196 \mathrm{~nm}$.

Near-UV Circular Dichroism of TTR at $0.02 \mathrm{mg} / \mathrm{mL}$. Wildtype TTR samples $(0.02 \mathrm{mg} / \mathrm{mL}, \mathrm{pH} 7,4.4$, and 2.0) were incubated at $25^{\circ} \mathrm{C}$ for $15 \mathrm{~h}$. Circular dichroism spectra were collected in a $10 \mathrm{~cm}$ quartz cell at $25^{\circ} \mathrm{C}$ using an Aviv model 62DS CD spectrometer employing a bandwidth of $0.5 \mathrm{~nm}$, a time constant of $2 \mathrm{~s}$, and a step size of $0.2 \mathrm{~nm}$. Thirty scans from 320 to $250 \mathrm{~nm}$ were recorded and averaged.

\section{RESULTS}

Under physiological conditions $(0.2-0.3 \mathrm{mg} / \mathrm{mL}, \mathrm{pH} 7$, $25^{\circ} \mathrm{C}$ ) wild-type TTR and the V30M and L55P FAPassociated TTR variants exhibit a tetrameric quaternary structure, as discerned by previous sedimentation equilibrium analysis after incubation at $25{ }^{\circ} \mathrm{C}$ for $24 \mathrm{~h}$ (29). Identical conditions were used in the sedimentation velocity studies presented here to determine the $s$ values for the TTR tetramers. The sedimentation velocity profiles of wild-type TTR at pH 7.0 show the movement of a single boundary across the cell (Figure 1A). The second moment analysis for the movement of the midpoint of the absorbance boundary $(r)$ vs time $(t)$ was used to obtain the apparent sedimentation coefficient, $s$, of $4.5 \mathrm{~S}$. This $s$ value corresponds to an $s_{20, \mathrm{w}}=4.1 \mathrm{~S}$, consistent with the TTR tetramer (28). Further analysis of the data using van Hold-Weischet global fitting also indicates the presence of one single species with $s_{20, \mathrm{w}}=4.2 \mathrm{~S}$ (Figure 1B,C). Sedimentation velocity analysis of V30M and L55P TTR after a $24 \mathrm{~h}$ incubation $\left(25^{\circ} \mathrm{C}\right)$ at $\mathrm{pH} 7$ also shows a single species with an $s_{20, \mathrm{w}}=$ 3.9 and $3.8 \mathrm{~S}$, respectively (data not shown).

Interestingly, sedimentation velocity analysis of L55P samples $(0.1$ and $0.2 \mathrm{mg} / \mathrm{mL})$ incubated at $37{ }^{\circ} \mathrm{C}$ for 2 months (pH 7.5) shows the presence of oligomeric species (protofilaments by EM characterization, see Figure 2) that sediment with an average $s$ value of $14 \mathrm{~S}$. Analysis of the sedimentation profiles by van Holde and Weischet analysis confirms the heterogeneity of the sample via the presence of a ladder of oligomeric species with $s$ values ranging from 2 to $23 \mathrm{~S}$, Figure 3. Analysis of the data using various methods and at various times during incubation reveals the absence of $>5 \%$ of the L55P tetramer after 2 months of incubation at $37{ }^{\circ} \mathrm{C}$. The L55P soluble protofilaments predominating in the sample are capable of binding congo red as discerned by the observed red shift of the congo red spectrum to $540 \mathrm{~nm}$ upon its addition to L55P TTR protofilaments (see Supporting Information). Sedimentation velocity analysis of a V30M TTR sample under the same conditions $\left(37^{\circ} \mathrm{C}, \mathrm{pH} 7.5\right)$ reveals the presence of a much smaller amount $(<10 \%$ by absorbance) of oligomeric intermediates (protofilaments); however, these oligomeric intermediates can be readily observed by electron microscopy. Unlike the L55P TTR case, tetrameric V30M TTR remains the predominant species in solution after a 2 month incubation at $\mathrm{pH} 7.5,37^{\circ} \mathrm{C}$, Figure 3B. It is not yet clear whether the V30M protofilaments will ultimately form amyloid fibrils; however, L55P is capable of amyloid fibril formation (protofilament assembly) under these conditions. The L55P and V30M oligomeric intermediates are operationally classified as protofilaments on the basis of their EM dimensions (47 $\pm 7 \AA$ diameter). These oligomers are 


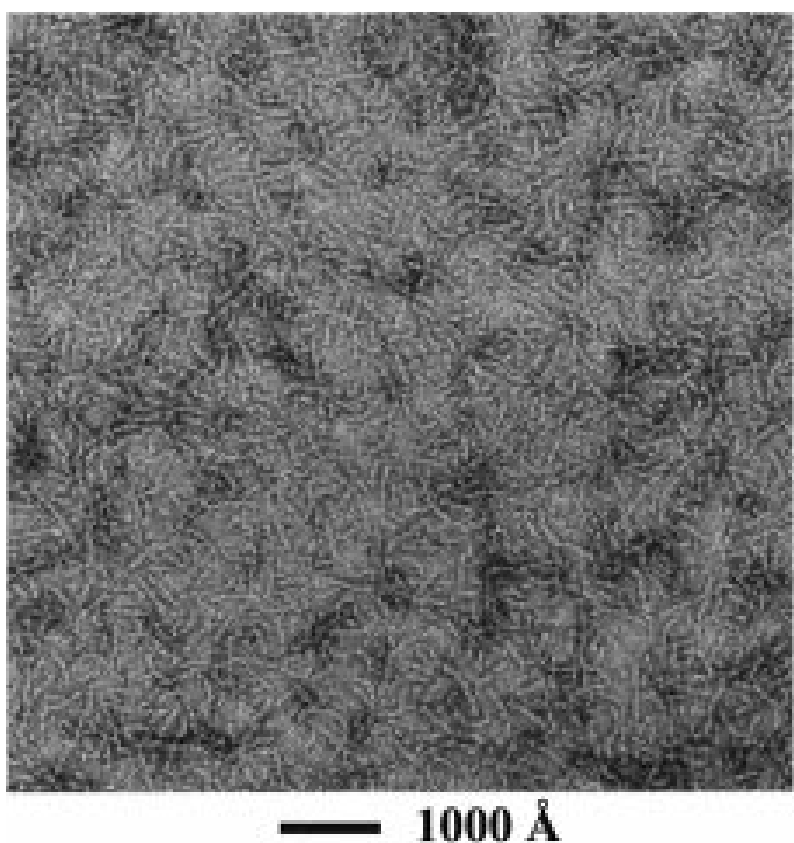

FIGURE 2: Electron micrograph of L55P TTR $(0.1 \mathrm{mg} / \mathrm{mL}, 100$ $\mathrm{mM} \mathrm{KCl}, \mathrm{pH} 7.5)$ incubated at $37^{\circ} \mathrm{C}$ for 2 months. The sample was prepared by allowing the solution to stand for $2 \mathrm{~min}$ on a carbon-coated grid before removing excess solution. The grid was then washed once with distilled water and once with $1 \%$ uranyl acetate before staining the protofilaments for 2 min with fresh $1 \%$ uranyl acetate. Magnification: 91000x. Scale bar $=1000 \AA$.

significantly smaller in diameter than the square 110-130 $\AA$ cross-sections of the amyloid fibrils observed by Blake composed of four protofilaments but are similar to the protofilaments composing the V30M amyloid fibrils that have a diameter of $40-50 \AA(33,34)$.

Sedimentation velocity analysis of L55P TTR ( $\mathrm{pH} 7.5)$ incubated for 7-15 days reveals that the amount of dissociated monomer formed is dependent on the incubation temperature, with more monomer observed the higher the incubation temperature (i.e., $37{ }^{\circ} \mathrm{C}>25{ }^{\circ} \mathrm{C}>4{ }^{\circ} \mathrm{C}$ ). The dissociation of the L55P tetramer to monomer at pH 7.5, 37 ${ }^{\circ} \mathrm{C}$ prior to the formation of the oligomeric intermediates strongly suggests that the monomer is the amyloidogenic intermediate and a precursor to amyloid protofilaments, even at physiological $\mathrm{pH}$. The tetramer to monomer dissociation for V30M under physiological conditions $\left(\mathrm{pH} 7.5,37^{\circ} \mathrm{C}\right.$ ) appears to be slow relative to L55P, as only trace amounts of monomer can be observed under conditions where the L55P monomer concentration is as high as $15 \%$. However, tetramer dissociation still takes place for $\mathrm{V} 30 \mathrm{M}$, as protofilaments formed from assembly of the monomeric amyloidogenic intermediate can be observed by electron microscopy after longer incubation periods ( 2 months) in low yield. It is likely that the rate of assembly of the V30M monomeric amyloidogenic intermediate $\left(\mathrm{pH} 7.5,37^{\circ} \mathrm{C}\right)$ is greater than the tetramer dissociation rate, making it difficult to observe significant amounts of the monomeric and oligomeric intermediates that precede protofilament formation, unlike the L55P case where the tetramer dissociation rate is higher.

Evaluation of wild-type TTR $(0.2 \mathrm{mg} / \mathrm{mL})$ by velocity studies at $\mathrm{pH} 5.5$ and 6.0 reveal the presence of a single species corresponding to the TTR tetramer with $s_{20, \mathrm{w}}=4.2$ $\mathrm{S}$. At $\mathrm{pH}$ 5.0, a second slower-moving boundary appears with an absorbance of less than $10 \%$ of the total. The major
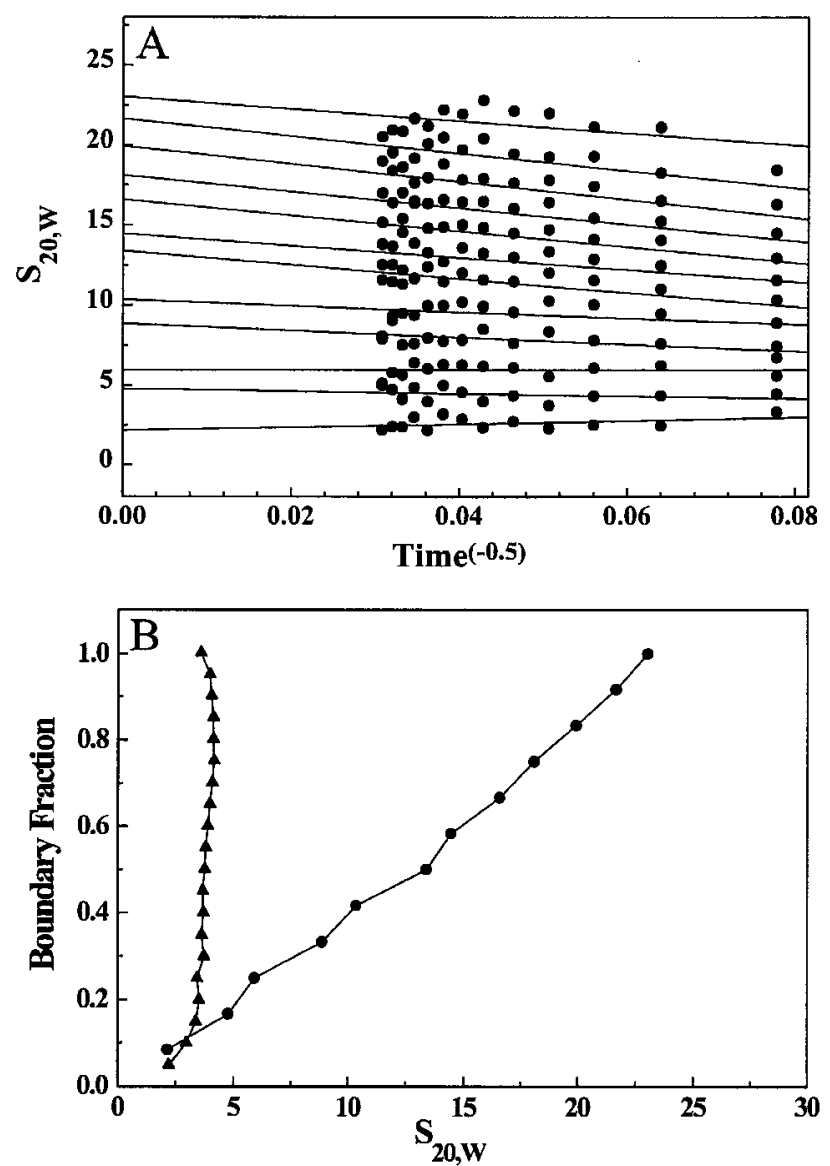

FIGURE 3: van Holde and Weischet analysis of L55P and V30M TTR $(0.1 \mathrm{mg} / \mathrm{mL}, \mathrm{pH} 7.5)$ incubated at $37{ }^{\circ} \mathrm{C}$ for 2 months. (A) van Holde and Weischt extrapolation plot of L55P illustrating the distribution of oligomeric quaternary structures. (B) $G(s)$ extrapolation plot of L55P (-) and V30M (A) illustrating the striking differences in the populations of the oligomeric intermediates observable by this method.

species (>90\%) sediments with an $s_{20, w}$ value of $4.1 \mathrm{~S}$, corresponding to TTR tetramer. The minor species has an $s_{20, \mathrm{w}}$ value of $1.2 \mathrm{~S}$, corresponding to monomeric TTR. A small fraction of oligomeric species is visible early in the velocity analysis, which likely results from association of the monomeric amyloidogenic intermediate (see below). Sedimentation studies on V30M and L55P TTR incubated at $\mathrm{pH} 6.0$ for $24 \mathrm{~h}$ indicate that both sediment as a homogeneous tetrameric species with $s$ values of 3.9 and 3.8 S, respectively. At pH 5.5 the L55P FAP variant exhibits extensive amyloid fibril formation unlike V30M, which exhibits modest amyloid fibril formation at $\mathrm{pH} 5.5$, or wildtype TTR, which does not exhibit amyloid fibril formation at $\mathrm{pH}$ 5.5.

Wild-type transthyretin $\left(0.2 \mathrm{mg} / \mathrm{mL}, 100 \mathrm{mM} \mathrm{KCl}, 37^{\circ} \mathrm{C}\right)$ exhibits maximal amyloid fibril formation at $\mathrm{pH} 4.4(72 \mathrm{~h})$ (29). Amyloid formation is also observed at the lower temperature of $25^{\circ} \mathrm{C}$ at $\mathrm{pH} 4.4$, albeit in lower yield (20$50 \%$ ). Sedimentation velocity analysis of TTR at $\mathrm{pH} 4.4$ after an incubation period of 2 days at $25^{\circ} \mathrm{C}$ is reported in a recent publication where inhibitors of TTR amyloid formation are evaluated (28). Under these conditions, TTR exists as a mixture of sedimenting species with $s$ values ranging from 1.6 to $6.3 \mathrm{~S}$ as discerned from the time derivative $g\left(s^{*}\right)$ analysis. The amyloid fibrils present in this solution quickly sediment to the bottom of the centrifuge cell, resulting in 

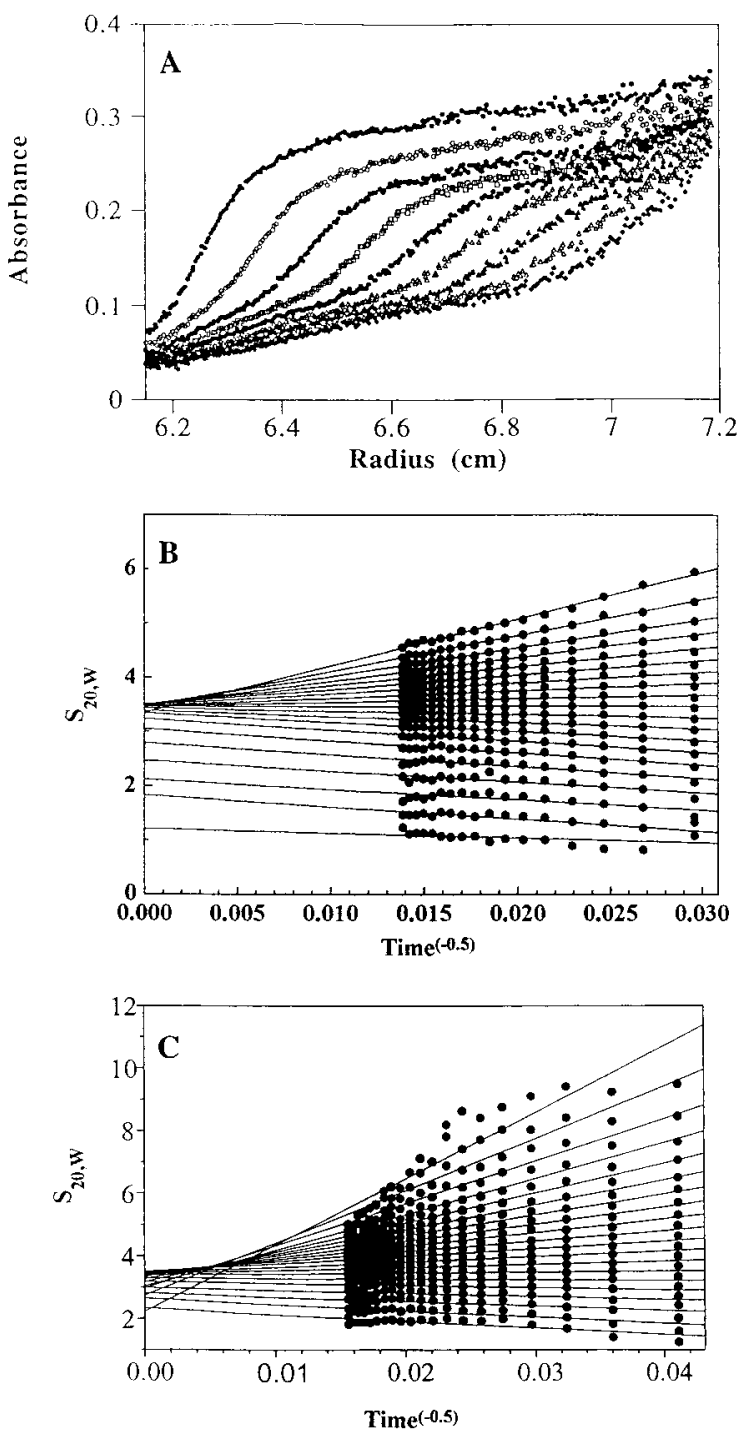

FIGURE 4: Sedimentation velocity studies of wild-type TTR $(0.2$ $\mathrm{mg} / \mathrm{mL}, \mathrm{pH} 4.4,25^{\circ} \mathrm{C}$ ). (A) Overlay of data sets recorded approximately 20 min apart. (B) van Holde and Weischet extrapolation plots. (C) Extrapolation plots of data sets which include early scans of the fast sedimenting species. These reveal the presence of intermediates as evident by the characteristic discontinuous decrease in the upper region of the boundary fraction.

the loss of $\approx 50 \%$ of the sample as large visible aggregates that can also be filtered ( $2 \mu \mathrm{m}$ filter) prior to sedimentation velocity studies. Velocity analysis of wild-type TTR quaternary structures $\left(\mathrm{pH} 4.4,25^{\circ} \mathrm{C}\right)$ remaining after filtration discloses the presence of predominately tetrameric TTR, $<10 \%$ of the monomeric amyloidogenic intermediate, and an even smaller amount $(<5 \%)$ of oligomeric quaternary structural intermediates, Figure 4, consistent with the conversion of the remainder into amyloid. Inclusion of early scans, during which the protein is pelleting during the velocity analysis reveals the presence of intermediates as evident by the characteristic discontinuous decrease in the upper region of the boundary fraction; see Figure 4C. It is difficult to observe the oligomeric intermediates for wild-type TTR due to the high activation barriers for the dissociation of the tetramer to the monomeric amyloidogenic intermediate relative to the activation barriers for assembly. Hence the intermediates do not build up in concentration because they are rapidly converted into amyloid fibrils via quaternary
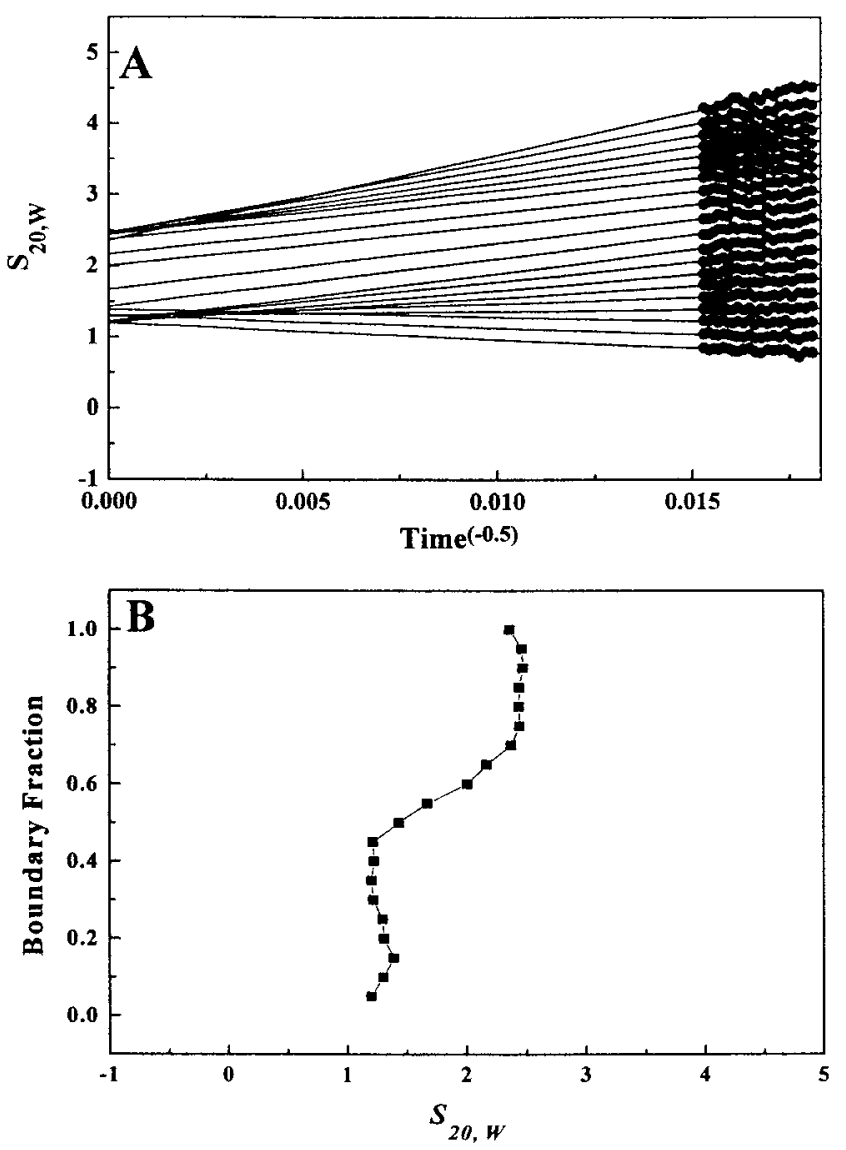

FIGURE 5: Sedimentation velocity analysis of wild-type TTR $(0.2$ $\mathrm{mg} / \mathrm{mL}$ ) incubated at $4{ }^{\circ} \mathrm{C}$ for $72 \mathrm{~h}$. (A) van Holde and Weischet extrapolation plot. (B) $G(s)$ distribution plot showing the presence of two species, monomer and possibly dimer.

structural intermediates. In an effort to slow the assembly process and thus observe the oligomeric intermediates at the expense of insoluble amyloid fibrils, TTR was incubated at $4{ }^{\circ} \mathrm{C}$ and evaluated by velocity experiments as a function of time.

An evaluation of wild-type TTR at $4{ }^{\circ} \mathrm{C}$ after incubation for $12,20,30$, and $72 \mathrm{~h}(\mathrm{pH} 4.4)$ reveals that tetrameric TTR dissociates and sediments as two major species with $s_{20, \mathrm{w}}$ values of 1.5 (monomer) and $2.5 \mathrm{~S}$ (uncharacterized quaternary structure, possibly dimer), but unexpectedly the monomer does not undergo self-assembly at $4{ }^{\circ} \mathrm{C}$; i.e., no oligomers or amyloid fibrils can be detected, Figure 5. The fraction of monomer observed increases with incubation time; e.g., we observe $65 \%$ monomer at $72 \mathrm{~h}$. These results suggest that it may not be sufficient to simply have TTR tetramer dissociation to a monomeric intermediate with nativelike tertiary structure; instead a tertiary structural rearrangement appears to be necessary as proposed earlier, which may not be energetically accessible at $4{ }^{\circ} \mathrm{C}(1,3,27)$. Another possibility for explaining the lack of fibril formation is that the amyloidogenic intermediate is formed but simply cannot self-assemble at $4{ }^{\circ} \mathrm{C}$. This seems unlikely as the V30M and L55P amyloidogenic intermediates can assemble into amyloid at $4{ }^{\circ} \mathrm{C}$ ( $\mathrm{pH} 4.4$; see below). Interestingly, amyloid fibril formation occurs quickly $(18-30 \mathrm{~h})$ upon transferring the 4 ${ }^{\circ} \mathrm{C}$ TTR solution containing predominantly monomeric wildtype TTR to a higher temperature $\left(37^{\circ} \mathrm{C}\right)$. The rate of wildtype TTR amyloid fibril formation is significantly higher for samples preincubated at $4{ }^{\circ} \mathrm{C}$ and then shifted to $37{ }^{\circ} \mathrm{C}$ 

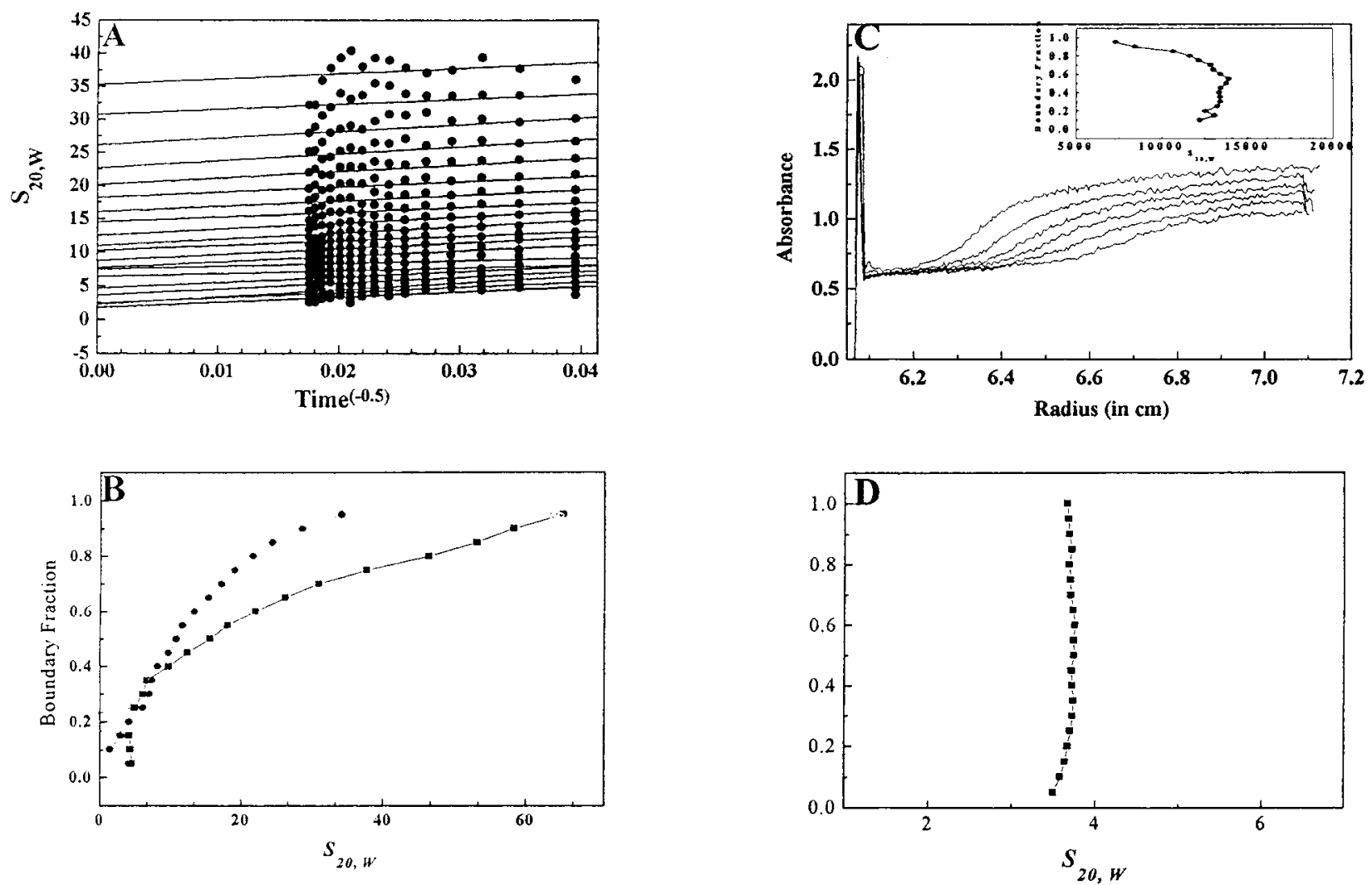

FIGURE 6: Sedimentation velocity studies of L55P and V30M TTR at pH $4.4\left(25^{\circ} \mathrm{C}\right)$. (A) van Holde and Weischet extrapolation plot of L55P after $12 \mathrm{~h}$. (B) $G(s)$ distribution plots of L55P TTR after $5(\boldsymbol{\square})$ and $12 \mathrm{~h}(\mathbf{O})$. (C) Sedimentation velocity profile of V30M TTR after $12 \mathrm{~h}$ at $3000 \mathrm{rpm}$. Inset shows the van Holde and Weischet analysis $G(s)$ distribution plot of the fast sedimenting species. (D) $G(s)$ plot of the species left in solution after sedimentation of V30M TTR at $3000 \mathrm{rpm}$, revealing mainly tetrameric TTR.

compared to samples incubated exclusively at $25{ }^{\circ} \mathrm{C}$ or 37 ${ }^{\circ} \mathrm{C}$, owing to the predominance of the tetrameric species in the latter samples (TTR incubated exclusively at 25 or 37 ${ }^{\circ} \mathrm{C}$ is only $\leq 5 \%$ monomeric at steady state).

The L55P and V30M FAP-associated TTR variants, like wild-type TTR, exhibit similar amyloid fibril formation at $\mathrm{pH} 4.4$, as discerned by light scattering and congo red binding $(23,24)$. Sedimentation velocity analysis of L55P TTR samples $\left(0.2 \mathrm{mg} / \mathrm{mL}\right.$ incubated at $25^{\circ} \mathrm{C}$ or $37^{\circ} \mathrm{C}$ for 2 days at $\mathrm{pH}$ 4.4) exhibit extensive fibril formation at both temperatures as discerned by rapid sedimentation of the amyloid component of the sample (80-90\% conversion of soluble L55P into amyloid at $37{ }^{\circ} \mathrm{C}$ ). In an attempt to characterize the amyloidogenic intermediates being formed by partial acid denaturation of L55P TTR, sedimentation velocity experiments were carried out on samples incubated for shorter time periods (5 and $12 \mathrm{~h}$ ) and at lower temperature $25{ }^{\circ} \mathrm{C}$. Analysis of the $5 \mathrm{~h}$ sample shows that most of the L55P TTR tetramer (63\%) had been converted to high molecular weight oligomeric intermediate species with $s$ values ranging from 3.9 to $66 \mathrm{~S}$; however, no amyloid fibrils were detected, Figure 6. Analysis of the $12 \mathrm{~h}$ L55P TTR $\left(25^{\circ} \mathrm{C}\right)$ samples reveals the presence of some amyloid fibrils (15-25\%) and a heterogeneous distribution of oligomeric intermediates remaining in solution with $s$ values ranging from 1.4 to 39 $\mathrm{S}$, Figure 6A,B. Tetrameric L55P TTR is not detectable $(\leq 5 \%)$ in the $12 \mathrm{~h}$ sample. Sedimentation analysis of L55P TTR samples incubated beyond $12 \mathrm{~h}$ reveal more amyloid fibril formation (up to $55 \%$ after 3 days, $25^{\circ} \mathrm{C}$ ), predominantly high-MW oligomeric intermediates in solution, and the absence of $>5 \%$ monomeric or tetrameric L55P TTR.
Analogous studies on V30M TTR reveal that the dissociated monomeric amyloidogenic intermediate rapidly forms amyloid fibrils, making it difficult to observe intermediates. Sedimentation velocity analysis of $0.2 \mathrm{mg} / \mathrm{mL}$ V30M incubated at $25^{\circ} \mathrm{C}$ for $15-20 \mathrm{~h}$ at $3000 \mathrm{rpm}$ show the presence of fast sedimenting species $(s=13000 \mathrm{~S})$, Figure 6C. However, analysis of the sample remaining in solution reveals predominantly tetrameric V30M TTR (Figure 6D), whereas no L55P tetramer can be detected under these conditions.

The rate of dissociation $(\mathrm{pH} 4.4)$ of the V30M tetramer to yield the monomeric amyloidogenic intermediate was slower than the rate exhibited by L55P, but not as slow as wild-type TTR, as discerned from time-dependent ultracentrifugation studies. As a consequence of the slower rate of formation of the V30M monomeric amyloidogenic intermediate and a relatively fast rate of fibril formation, it is difficult to observe the oligomeric amyloidogenic intermediates. In an attempt to slow the self-association process leading to V30M amyloid fibrils, the temperature was reduced to $4{ }^{\circ} \mathrm{C}$ to facilitate observation of the oligomeric intermediates. High-MW oligomeric intermediates can be observed as the dominant species in solution when V30M TTR $(0.2 \mathrm{mg} / \mathrm{mL}$, $\mathrm{pH}$ 4.4) is incubated at $4{ }^{\circ} \mathrm{C}$; ultimately amyloid fibril formation is observed but only after extended incubation periods or a temperature jump to $25{ }^{\circ} \mathrm{C}$. Sedimentation velocity studies of $4{ }^{\circ} \mathrm{C}$ samples incubated for $72 \mathrm{~h}$ and evaluated by van Hold-Weischet analysis reveals the presence of a series of oligomeric intermediates with $s$ values ranging from 10 to $140 \mathrm{~S}$, Figure 7A,B. Analysis of $95 \%$ of the sedimenting boundaries shows that no TTR monomer 

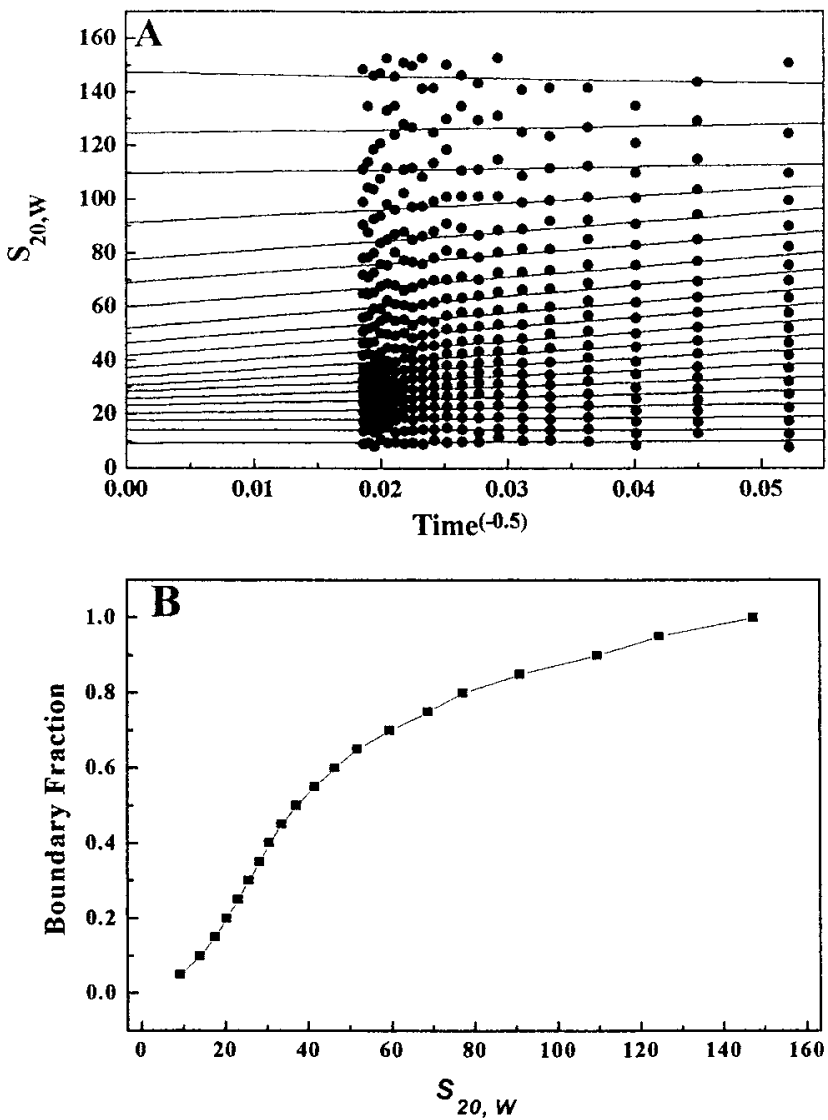

FIGURE 7: Sedimentation velocity analysis of V30M TTR $(0.2 \mathrm{mg} /$ $\mathrm{mL}$ ) incubated at $4{ }^{\circ} \mathrm{C}$ for $72 \mathrm{~h}(\mathrm{pH} 4.4)$. (A) Extrapolation plot. (B) $G(s)$ plot illustrating the oligomeric intermediates present.

or tetramer can be detected under these conditions. This result is distinctly different from that exhibited by wild-type TTR, where dissociation of the tetramer to monomer is observed, but no assembly or amyloid fibril formation occurs at $4{ }^{\circ} \mathrm{C}$. The fact that the V30M and L55P amyloidogenic intermediate can assemble into amyloid at $4{ }^{\circ} \mathrm{C}(\mathrm{pH} 4.4)$ indicates that the barrier for the rearrangement of the tertiary structure can be overcome at $4{ }^{\circ} \mathrm{C}$ for V30M and L55P, but not for wild-type TTR. In summary, the L55P and V30M oligomeric intermediates appear to be formed by the self-assembly of the monomeric amyloidogenic intermediate, consistent with the presence of monomer at early stages and the broad MW distribution observed at later stages, especially for L55P at $25{ }^{\circ} \mathrm{C}$. None of the intermediates appear to predominate; rather a ladder of quaternary structures is observed during amyloid fibril formation.

Amyloid formation is not observed below pH 3 for wildtype TTR, even after an incubation period of 3 days at 37 ${ }^{\circ} \mathrm{C}$, as judged by the absence of light scattering (29). SDSPAGE analysis of the quaternary structure at $\mathrm{pH} 3$ reveals that wild-type TTR and the TTR variants V30M and L55P run exclusively as a monomeric band in the gel at this $\mathrm{pH}$, consistent with either a monomeric structure or a higher order quaternary structure that is SDS sensitive. Attempts to study the quaternary structure of wild-type TTR at $\mathrm{pH} 3.0$ by the sedimentation equilibrium methods failed due to the apparent aggregation of TTR during the runs (29). A sedimentation velocity study of wild-type TTR at $\mathrm{pH} 3$ coupled with van Hold and Weischet analysis identifies the presence of multiple sedimenting species with $s$ values ranging from 3
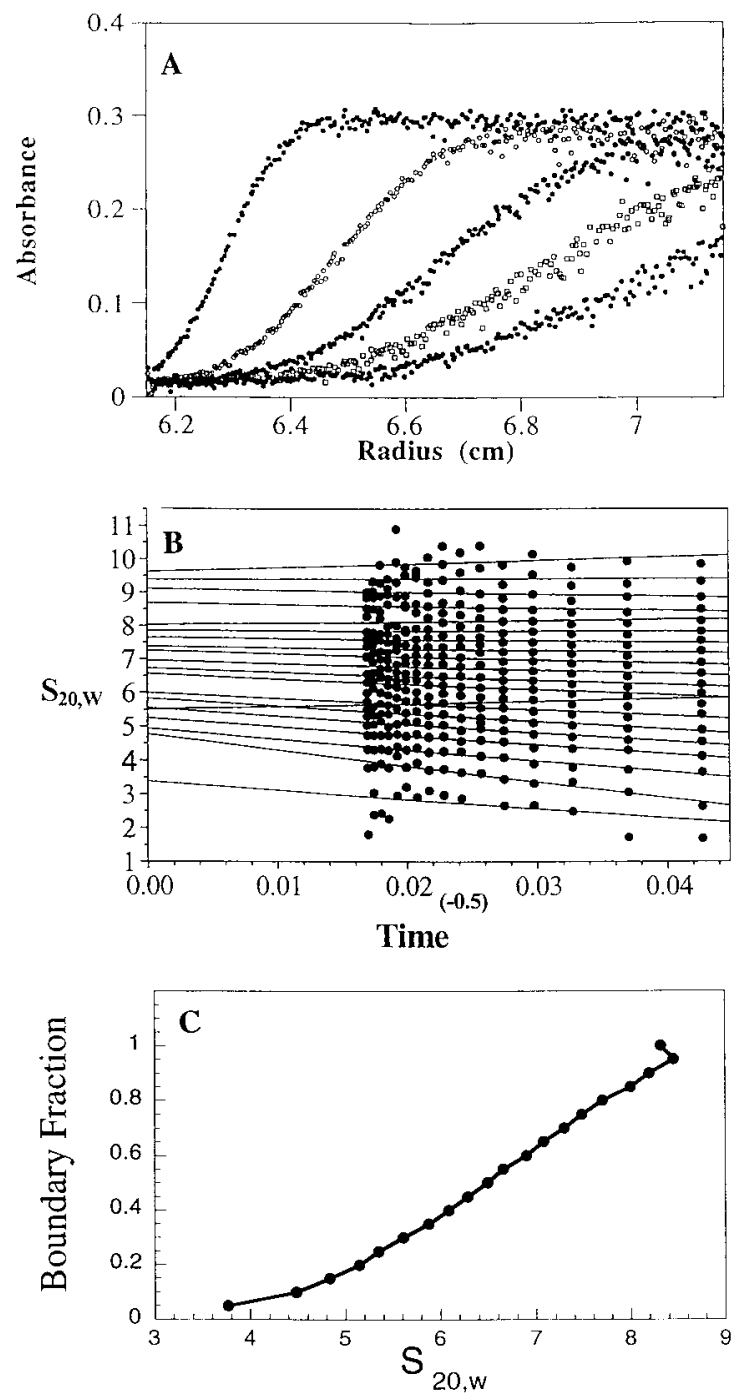

FIGURE 8: Sedimentation velocity studies on wild-type TTR $(0.2$ $\mathrm{mg} / \mathrm{mL}$ ) incubated at $\mathrm{pH} 3.0$. (A) Overlay of data sets approximately 15 min apart. (B) van Holde and Weischet extrapolation plot showing the oligomeric quaternary structures present. (C) $G(s)$ distribution plot of wild-type TTR showing the quaternary structural heterogeneity.

to $9.0 \mathrm{~S}$, Figure 8 , consistent with an assembled state at $\mathrm{pH}$ 3. Of significance, the resulting quaternary structures have a lower MW distribution and are incapable of forming amyloid fibrils. The quaternary structural distribution is very different from what we observe for wild-type, V30M, and L55P TTR at $\mathrm{pH} 4.4$, where a ladder of intermediates having $s$ values as high as $140 \mathrm{~S}$ are observed under amyloid fibril forming conditions.

Previous far-UV CD studies indicate that wild-type TTR still exhibits a high content of $\beta$-sheet structure at $\mathrm{pH} 2$; however, the tertiary structure is non-native on the basis of near-UV CD and fluorescence data. Owing to these results, we proposed that wild-type TTR adopted an A-state conformation, similar to that of a molten globule at $\mathrm{pH} 2$ with $\mathrm{KCl}$ present (100 mM) (29). However, sedimentation velocity analysis at $\mathrm{pH} 2(0.2 \mathrm{mg} / \mathrm{mL})$ revealed that wild-type TTR sediments with an apparent $s$ value (7.2 S), much higher than that of a monomer (1.6 S). Furthermore, the observed boundaries are very broad, indicating that more than one species exists at this $\mathrm{pH}$. The van Hold and Weischet analysis for wild-type TTR and the TTR variants confirms the 

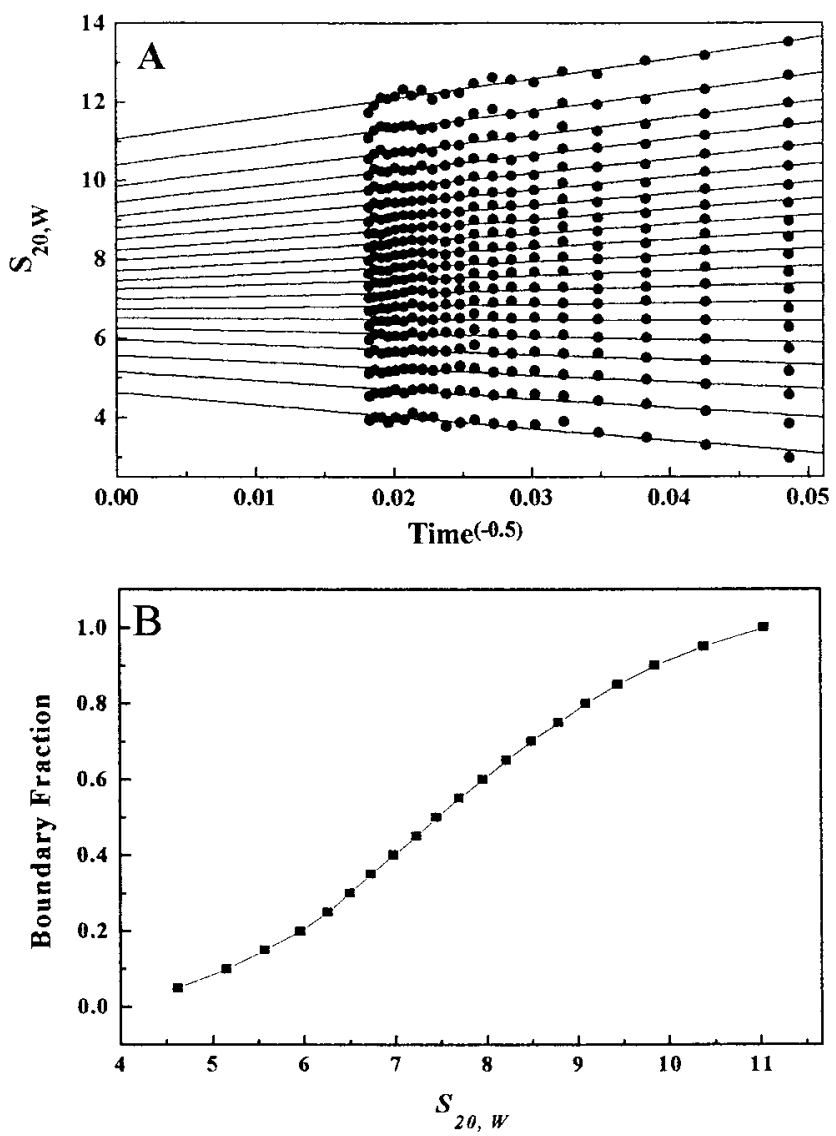

FIGURE 9: Sedimentation velocity analysis of L55P TTR at $\mathrm{pH}$ $2.0(0.2 \mathrm{mg} / \mathrm{mL})$. (A) van Holde and Weischet extrapolation plots on data sets taken 9 min apart, illustrating the heterogeneity of the sample. (C) $G(s)$ plot shows the presence of multiple quaternary structures.

presence of multiple species and shows the distribution of species with $s$ values ranging from 3 to $8 \mathrm{~S}$, from 1 to $9 \mathrm{~S}$, and from 4 to $12 \mathrm{~S}$ for wild-type, V30M, and L55P TTR, respectively, at $\mathrm{pH} 2$. Representative data processed by the van Holde and Weischet analysis for L55P TTR are shown in Figure 9. These sedimenting species correspond to a molecular weight range of 14000 to approximately 260000 , suggesting that monomeric TTR is the building block of this array of species. Recent time course data collected at $\mathrm{pH}$ 2.0 and 3.0 using atomic force microscopy and electron microscopy confirm that wild-type TTR assembles into small oligomeric species that do not go on to form amyloid fibrils, even after incubation periods of more than 1 month at 25 ${ }^{\circ} \mathrm{C}$. Previous biophysical studies on wild-type TTR as a function of $\mathrm{pH}$ indicate that the difference in assembly at $\mathrm{pH} 4.4$ (amyloid) and at $\mathrm{pH} 2.0$ (assembled A-state) results from the differences in the tertiary structures of the TTR monomer precursors (29).

Studies on acid molten globules (A-states) suggest that anion binding induces collapse of proteins at low $\mathrm{pH}$ (5153). Wild-type TTR exhibits a far-UV CD spectrum with a minimum around $200 \mathrm{~nm}$ at $\mathrm{pH} 2$ in the absence of added salt, consistent with a random coil conformation (29). Sedimentation velocity experiments of wild-type TTR at $\mathrm{pH}$ 2.0 in the absence of salt were carried out to improve our understanding of the mechanism of this salt induced structural change. The observed boundaries were broad, probably due to diffusion of monomeric TTR. A direct fitting of the data
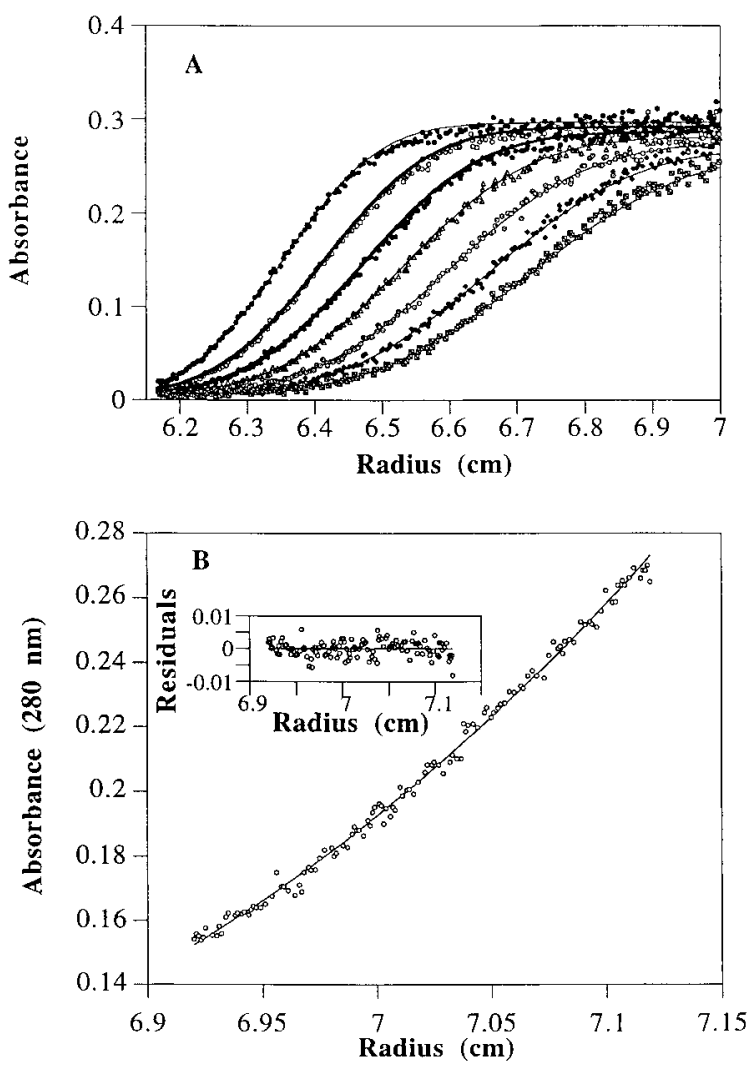

FIGURE 10: Sedimentation velocity profiles of wild-type TTR $(0.2$ $\mathrm{mg} / \mathrm{mL}$ ) at $\mathrm{pH} 2.0$ in the absence of salt. (A) Overlay of data sets (represented by different symbols) recorded approximately $30 \mathrm{~min}$ apart. The solid lines represent direct fitting of the data to a single ideal species by the Svedberg program. (B) Sedimentation equilibrium data fit to a single ideal species model (solid line) affording a molecular weight of 14000 . The Insert shows the residual difference between the experimental data and the fit for each point.

using the Svedberg program gives a good fit of the data to a single ideal species model (Figure 10A), yielding an $s$ value of $1.23 \mathrm{~S}$ and a diffusion coefficient $(D)$ of $7.90 \mathrm{~F}$ affording a molecular weight of $14.9 \times 10^{3}(\mathrm{eq}$ II), in good agreement with the expected molecular weight of monomeric TTR (13.8 $\times 10^{3}$ ). Sedimentation equilibrium analysis was also performed at $\mathrm{pH} 2$ in the absence of salt, giving an excellent fit to a single ideal species model having a molecular weight of 14000 , Figure 10B. Sedimentation velocity experiments for V30M and L55P TTR at pH 2.0 in the absence of salt also show that these FAP variants sediment as a single species with an $s$ values corresponding to that of the monomer. These data and previous far-UV CD data demonstrate conclusively that, in the absence of salt, wild-type, V30M, and L55P TTR exist as an unfolded monomer at $\mathrm{pH}$ 2 . The addition of salt induces linked $\beta$-sheet structure formation (CD minimum is shifted to $214 \mathrm{~nm}$ ) and monomer self-association, affording a distribution of oligomeric species which have quaternary structures smaller than those observed under amyloid fibril forming conditions ( $\mathrm{pH}$ 5-3.9).

To investigate the effect of TTR concentration on amyloid fibril formation $(\mathrm{pH} 4.4)$ and oligomeric A-state formation (pH 2), sedimentation experiments were carried out at a wildtype TTR concentration an order of magnitude below physiological $\left(0.02 \mathrm{mg} / \mathrm{mL}\right.$ at $\left.25{ }^{\circ} \mathrm{C}\right)$. At $\mathrm{pH} 4.4$, the sedimentation profiles show two broad boundaries, Figure 11. The Svedberg analysis gives a reasonable fit to the twospecies model (slow moving boundary, $s_{1}=1.81 \mathrm{~S}, D_{1}=$ 


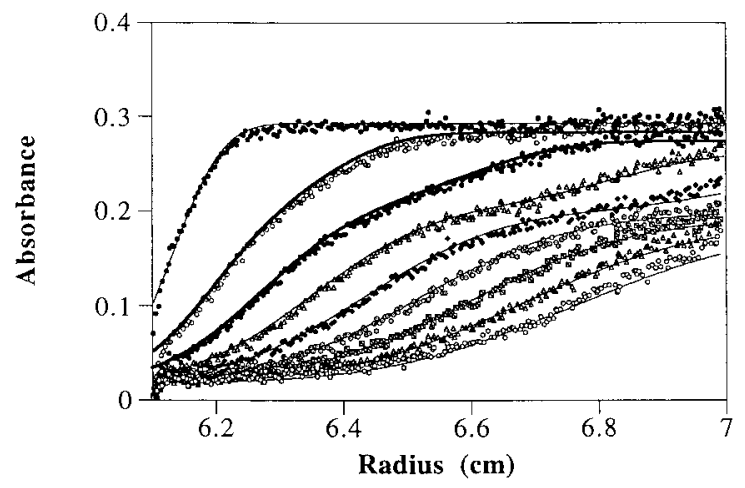

FIGURE 11: Sedimentation velocity studies of wild-type TTR (0.02 $\mathrm{mg} / \mathrm{mL})$ at $\mathrm{pH} 4.4\left(25{ }^{\circ} \mathrm{C}\right)$. Overlay of data sets recorded approximately $30 \mathrm{~min}$ apart are presented. The solid lines through the data represent the direct fit of the absorbance data to a twospecies model using the Svedberg software.

$16.62 \mathrm{~F}$; fast moving boundary, $s_{2}=4.73 \mathrm{~S}, D_{2}=13.2 \mathrm{~F}$ ) corresponding to roughly $75 \%$ monomer and $25 \%$ tetramer on the basis of initial absorbance values. Note that the diffusion coefficients are much higher than expected for a single species in each case. This likely arises from the heterogeneity of each of the boundaries; i.e., each boundary may contain more than one species. An analysis of earlier sedimentation scans reveals the presence of a minor population of species with even higher $s$ values, indicating assembly of the monomeric amyloidogenic intermediate $(0.02 \mathrm{mg} / \mathrm{mL})$. This result is different from the sedimentation data derived from wild-type TTR at physiological concentration $(0.2 \mathrm{mg} /$ $\mathrm{mL}$ ), where predominantly tetramer and less than $5 \%$ higher order oligomers are observed at $\mathrm{pH} 4.4$, owing to the rapid sedimentation of over half the sample as amyloid fibrils.

The sedimentation of a low concentration $(0.02 \mathrm{mg} / \mathrm{mL})$ of wild-type TTR at $\mathrm{pH} 2$ exhibits a major species having an $s$ value around $1.4 \mathrm{~S}$, corresponding to monomer. Minor species with $s$ values around $3.0 \mathrm{~S}$ are also present, but in low quantities. At physiological concentration $(0.2 \mathrm{mg} / \mathrm{mL})$, multiple oligomeric species with $s$ values ranging from 4 to $8 \mathrm{~S}$ are observed, demonstrating that self-association of the acid-denatured state of TTR is concentration-dependent, as expected.

The effect of low temperature $\left(4^{\circ} \mathrm{C}\right)$ on TTR denaturation at $\mathrm{pH} 2$ was also examined. Sedimentation velocity experiments carried out at physiological concentration $(0.2 \mathrm{mg} /$ $\mathrm{mL}$ ) exhibit two broad boundaries. van Holde and Weischet analysis reveals that the major species have $s \approx 1$ and $3 \mathrm{~S}$ $\left(s_{20, \mathrm{w}}=1.2\right.$ and $\left.3.9 \mathrm{~S}\right)$, corresponding to TTR monomer and tetramer, respectively. This is different than the results from the velocity experiments on TTR at $25^{\circ} \mathrm{C}(\mathrm{pH} 2.0)$, which identify oligomeric species with an average $s$ value of 7.2 $\mathrm{S}$. These results suggest that the hydrophobic effect plays an important role in the self-association of the A-state at $\mathrm{pH}$ 2.0 , as a decrease in temperature typically attenuates the hydrophobic effect. The activation barrier for denaturation may also play a role.

\section{DISCUSSION}

The studies presented here employ analytical ultracentrifugation methods for characterizing the quaternary structure of the intermediates on the wild-type, V30M, and L55P TTR acid denaturation pathways, especially those that self- assemble into protofilaments or amyloid fibrils. These studies were carried out at physiological concentration $(0.2 \mathrm{mg} / \mathrm{mL})$ and at lower concentration $(0.02 \mathrm{mg} / \mathrm{mL})$, which is very close to the concentration used in previous fluorescence studies $(0.01 \mathrm{mg} / \mathrm{mL})$. The analytical ultracentrifugation results reveal that wild-type TTR exists as a tetramer from $\mathrm{pH} 7$ to pH $5.1(0.2 \mathrm{mg} / \mathrm{mL})$. From $\mathrm{pH} 5.0$ to 3.9 the wild-type tetramer slowly dissociates to a monomeric amyloidogenic intermediate that self-associates into amyloid fibrils under physiological conditions $\left(0.2 \mathrm{mg} / \mathrm{mL}, 37^{\circ} \mathrm{C}\right)$, with maximum fibril formation observed at $\mathrm{pH} 4.4(72 \mathrm{~h})$ (29). Sedimentation velocity analysis of a $0.2 \mathrm{mg} / \mathrm{mL}$ TTR solution incubated for 2 days at $\mathrm{pH} 4.4\left(25^{\circ} \mathrm{C}\right)$ discloses the presence of $\approx 50 \%$ amyloid fibrils. The remainder is predominately tetrameric TTR, $<10 \%$ of the monomeric amyloidogenic intermediate, and an even smaller amount $(<5 \%)$ of oligomeric quaternary structural intermediates, Figure 4. The importance of the conformationally altered monomeric amyloidogenic intermediate is clearly evident when wild-type TTR is incubated at $4{ }^{\circ} \mathrm{C}(\mathrm{pH} 4.4)$, which mediates tetramer dissociation to a monomer that is not competent to assemble into amyloid, presumably because the required tertiary structural changes do not occur at this temperature. When the $4{ }^{\circ} \mathrm{C}$ wild-type sample is jumped to $37{ }^{\circ} \mathrm{C}$, amyloid fibril formation occurs to a greater extent than in the case of wild-type samples incubated exclusively at $37{ }^{\circ} \mathrm{C}$, owing to the increased fraction of monomeric amyloidogenic intermediate present in samples incubated first at 4 and then at $37{ }^{\circ} \mathrm{C}$.

The self-assembly of the monomeric amyloidogenic intermediate into quaternary structural intermediates is much more apparent in the case of the familial amyloid polyneuropathy-associated variants V30M and especially L55P, Figure 6. The ease of intermediate detection decreases when comparing L55P to V30M to wild-type TTR owing to the higher steady-state concentration of the monomeric amyloidogenic intermediate in those cases where the intermediates are readily observable, e.g., L55P. The difference in the concentrations of the monomeric amyloidogenic intermediate seem to be caused by the rates of amyloid assembly relative to the rate of monomeric amyloidogenic intermediate formation (tetramer dissociation along with tertiary structural changes). The faster the rate of tetramer dissociation is relative to the rates governing the assembly of the monomeric amyloidogenic intermediate, the easier intermediate detection is. The importance of the formation of the monomeric amyloidogenic intermediate is also consistent with earlier data from our laboratory showing that several of the single site amyloidogenic variants that cause FAP behave similarly, in that they destabilize tetrameric TTR in favor of the monomeric amyloidogenic intermediate $(23,24)$. Ultracentrifuge data described within also imply that the rate of formation of the monomeric amyloidogenic intermediate for L55P is fast relative to the assembly rate under acidic conditions; hence, a ladder of quaternary structural intermediates are prevalent and easily observed for L55P by sedimentation velocity studies, Figure 6A,B. The V30M tetramer appears to dissociate to the monomeric amyloidogenic intermediate more slowly relative to the assembly rate; hence, the oligomeric intermediates are harder to observe, but they can be readily observed by electron microscopy. The suggestion that the activation barriers are lowered for the dissociation of the L55P and V30M TTR 
pH 7.5

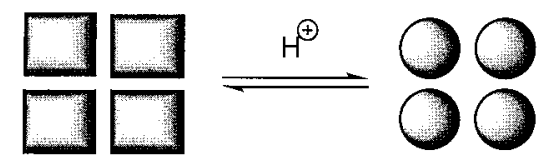

Rearranged

Native Tetrameric TTR Tetrameric TTR
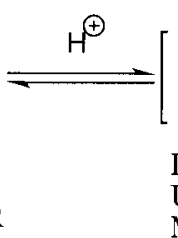

\begin{abstract}
Dimeric TTR Unstable Under Most Conditions
\end{abstract}

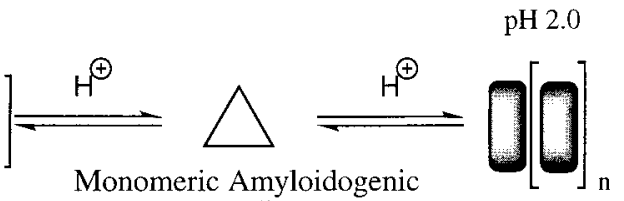

Monomeric Amyloidogenic Associated A-State

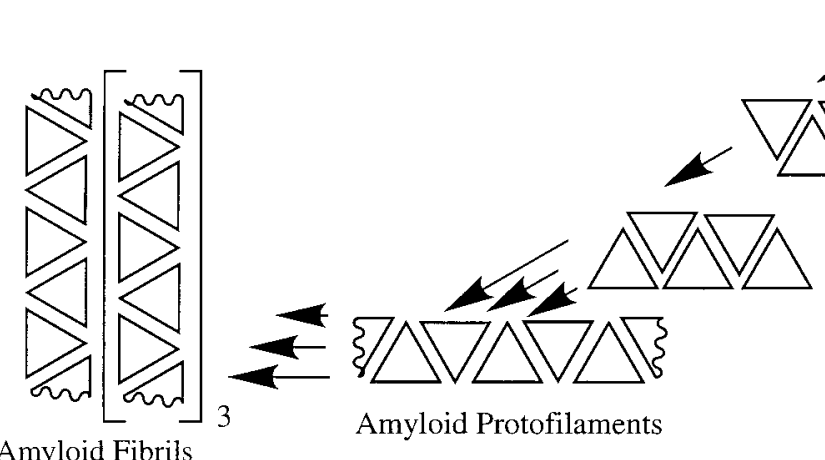

Amyloid Fibrils

FIGURE 12: Acid denaturation pathway of TTR accommodating all of the biophysical data recorded to date.

tetramer to the monomeric amyloidogenic intermediate is also strongly supported by the observation that V30M and L55P TTR undergo the formation of the monomeric amyloidogenic intermediate and assemble into protofilaments at $\mathrm{pH} 7.5$ (37 ${ }^{\circ} \mathrm{C}$ ), unlike wild-type TTR which remains normally folded, Figures 2 and 3. The fact that wild-type TTR cannot form the monomeric amyloidogenic intermediate under these conditions supports other evidence for the importance of this intermediate for amyloid fibril formation $(1,3)$.

The physiological relevance of the thermally induced partial denaturation $\left(37^{\circ} \mathrm{C}, \mathrm{pH} 7.5\right)$ of $\mathrm{V} 30 \mathrm{M}$ and L55P that yields amyloid protofilaments is not clear at the moment owing to the amount of time this process takes (weeks) relative to the $2-3$ day half-life of TTR in human plasma. Also, at the present time it is uncertain as to whether the V30M protofilaments will ever become mature amyloid fibrils; however, this process can be very slow as in the $\mathrm{A} \beta$ case. The half-life of the protein becomes irrelevant if the fibril can simply grow in the extracellular space as individuals age owing to the low but finite concentration of the monomeric amyloidogenic intermediate. Slow growth is feasible if the fibril is resistant to proteolytic degradation, which seems to be the case.

The data within and previous data present even stronger evidence that the partial acid denaturation of wild-type, V30M, and L55P TTR leads to a conformationally altered monomeric amyloidogenic intermediate that self-assembles into amyloid fibrils through a ladder of quaternary structural intermediates. The activation barriers for the formation of the monomeric amyloidogenic intermediate appear to increase in the order of L55P $<$ V30M $<$ wild-type TTR, employing either acid or thermal denaturation on the basis of time-dependent analysis $(23,24,29)$. These results are strictly consistent with recent kinetic GdnHCl-mediated denaturation studies of wild-type, V30M, and L55P TTR, demonstrating that V30M and L55P denature more quickly than wild-type TTR $(30,31)$. Hence, the FAP-associated mutations appear to kinetically and thermodynamically destabilize the TTR tetramer, thus increasing the steady-state concentration of the amyloidogenic intermediate, predispos- ing individuals with these mutations to amyloid fibril formation and disease. This logic is consistent with the age of amyloid disease onset, which is earliest for the most destabilized FAP variant (L55P disease onset $\approx 20$ years of age), followed by the FAP variant of intermediate stability (V30M, disease onset $\approx 35$ years of age), followed by the wild-type protein involved in the senile amyloid disease SSA (wild-type TTR, disease onset $\approx 80$ years of age). Comprehensive studies on the denaturation rates of various transthyretin sequences has commenced in the laboratory to understand how large the differences in denaturation rates are when acid or thermal denaturation is employed.

Sedimentation velocity data in conjunction with sedimentation equilibrium results clearly demonstrate the oligomeric nature of the nonamyloidogenic quaternary structures formed below pH 3 (100 mM KCl, $50 \mathrm{mM} \mathrm{HOAc})$, indicating that the TTR monomer is also capable of self-assembling into soluble A-state like aggregates. However, transthyretin denatured below pH 3 does not form amyloid, most likely owing to the tertiary structural disorder in the monomeric precursors to the aggregates (evident from inspection of the fluorescence denaturation curves) (29).

The analytical ultracentrifugation data presented here require that we reevaluate some prior spectroscopic data collected at physiological concentration, as a result of our improved understanding of the TTR quaternary structure changes as a function of $\mathrm{pH}$. However, through fortunate circumstances, conclusions drawn about the monomeric species formed below pH 5 are largely correct (29). The modified acid-denaturation pathway of TTR accommodating the present and prior results is presented in Figure 12. In previous studies, we employed SDS-PAGE, far- and nearUV CD, fluorescence, and sedimentation equilibrium methods to characterize the acid denaturation pathway of TTR (29). The SDS-PAGE method used previously to evaluate the TTR quaternary structure is excellent for evaluating the amount of native tetramer present (runs on the gel as a dimer) relative to other forms of TTR but is not faithful at representing the amount of monomer present because ag- 


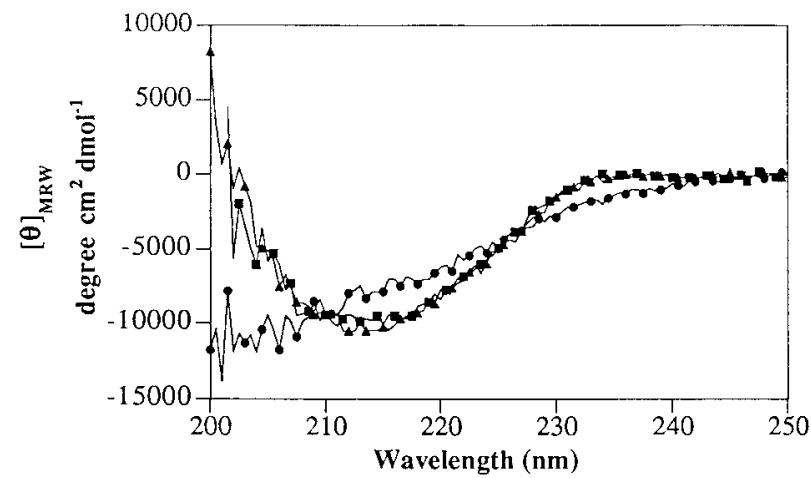

FIGURE 13: Far-UV CD spectra of TTR $(0.02 \mathrm{mg} / \mathrm{mL}, 100 \mathrm{mM}$ $\left.\mathrm{KCl}, 25^{\circ} \mathrm{C}\right)$ at $\mathrm{pH} 7.0(\boldsymbol{\Delta}), 4.4(\mathbf{\square})$, and $2.0(\bullet)$.

gregated forms of TTR dissociate to monomer in an SDS gel.

The interpretation of the far- and near-UV CD data collected at physiological concentration $(0.2 \mathrm{mg} / \mathrm{mL})$ at $\mathrm{pHs}$ $<5$ is complicated by the heterogeneity of the soluble TTR (29). The nativelike $\beta$-sheet far-UV CD spectral properties exhibited by TTR over the amyloid forming $\mathrm{pH}$ range represents an average property of all the TTR quaternary structural species in solution, including monomeric TTR. The current analytical ultracentrifugation data on wild-type, V30M, and L55P TTR at low concentration $(0.02 \mathrm{mg} / \mathrm{mL})$ demonstrates that monomeric TTR is the major species present at $\mathrm{pH}$ 4.4. As a result, we rerecorded far-UV CD data at this lower concentration, which demonstrates that the extent of $\beta$-sheet structure is not significantly changed in the transition from tetrameric TTR ( $\mathrm{pH} 7)$ to the monomeric amyloidogenic intermediate formed at $\mathrm{pH} 4.4$, Figure 13, consistent with our original interpretation (29).

The far-UV CD spectrum of wild-type TTR at $\mathrm{pH} 2.0$ under physiological salt conditions indicates that the protein at low concentration $(0.02 \mathrm{mg} / \mathrm{mL})$ exists as a mixture of $\beta$-sheet and random coil conformations, Figure 13. Sedimentation velocity data recorded under identical conditions at $\mathrm{pH} 2$ suggest that the wild-type TTR monomer is the major species, along with minor oligomeric products derived from the self-assembly of TTR monomer. It is possible that the TTR monomer under these conditions is largely unfolded, while the existence of the assembled form of TTR gives rise to the observed $\beta$-sheet signal; however, this seems unlikely on the basis of the numerous monomeric A-states characterized. Furthermore, the interpretation that the tertiary structure is less well-defined at $\mathrm{pH} 2$ is also consistent with the fluorescence data (29).

The original interpretation of the near-UV CD spectral changes exhibited by wild-type TTR $(0.2 \mathrm{mg} / \mathrm{mL})$ over the amyloid forming $\mathrm{pH}$ range is also complicated by selfassembly of the monomeric amyloidogenic intermediate (29). Near-UV CD data recorded at a lower TTR concentration $(0.02 \mathrm{mg} / \mathrm{mL})$ as a function of $\mathrm{pH}$ (where self-assembly is minimal) reveal a modest tertiary structural change upon formation of the monomeric amyloidogenic intermediate. An intensity decrease is observed in the near-UV CD spectrum upon changing the $\mathrm{pH}$ from 7 to 4.4 or 3.9 ; however, the maxima do not change in frequency, Figure 14. Hence, we can conclude that the formation of the amyloidogenic intermediate does not involve marked tertiary structural changes, at least in the region of $\operatorname{Trp} 79$ in the short helix,

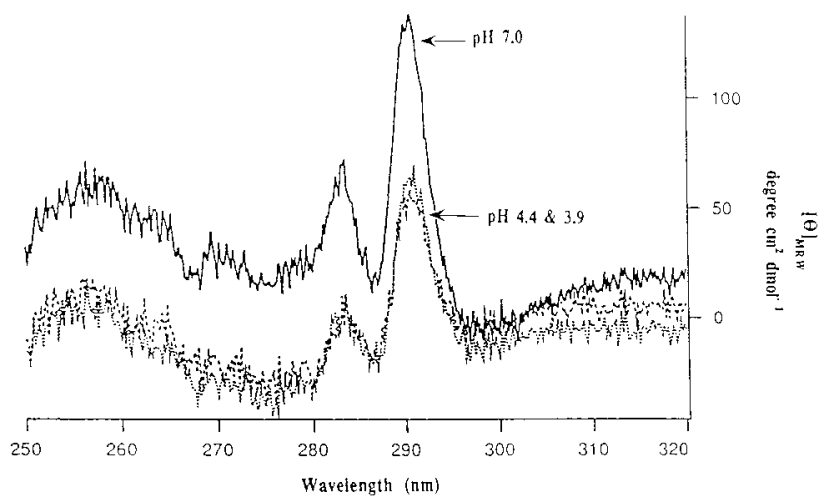

FIGURE 14: Near-UV CD spectra of wild-type TTR $(0.02 \mathrm{mg} / \mathrm{mL})$ at $\mathrm{pH} 7.0,4.4$, and 3.9 recorded in a $10 \mathrm{~cm}$ quartz cell using the conditions described in Materials and Methods.

consistent with our original interpretation (29). We have shown that Trp 79 is the primary contributor to the nearUV CD spectrum in TTR, via Trp to Phe mutations that have not yet been reported. Other data not outlined here support a tertiary structural rearrangement occurring remotely from Trp 79 in the C-strand-loop-D-strand region (1).

The original interpretation of the wild-type TTR fluorescence denaturation curves as a function of $\mathrm{pH}$ is fully supported by the analytical ultracentrifuge results reported within (29). The fluorescence experiments were carried out at $0.01 \mathrm{mg} / \mathrm{mL}$, where self-assembly is not a significant problem below $\mathrm{pH} 5$. Sedimentation experiments were attempted at $0.01 \mathrm{mg} / \mathrm{mL}$ where the fluorescence measurements were made; however, due to the absorption optics used, a wild-type TTR concentration of $0.02 \mathrm{mg} / \mathrm{mL}$ was the lowest concentration where a good signal-to-noise ratio could still be obtained. The Trp-based fluorescence denaturation curves for WT, V30M, and L55P TTR exhibit a plateau region over the amyloid forming $\mathrm{pH}$ range, suggesting the buildup of a monomeric intermediate(s) with an altered tertiary structure, differing from the tertiary structure observed at $\mathrm{pH} 7$ and $\mathrm{pH} 2$. The minor fluorescence contribution from the partially assembled TTR amyloidogenic intermediate $(<5 \%)$ is not expected to change the spectral interpretation because fluorescence measurements are relatively insensitive to quaternary structural changes, unless the fluorophores are located at the interface, which is not the case here (54).

The difference between the assembly of the TTR monomer formed at $\mathrm{pH} 4.4$ and $\mathrm{pH} 2$ is that the tertiary structure in the monomer formed at $\mathrm{pH} 4.4$ facilitates self-association into a high-MW insoluble amyloid fibril, while the lessordered low-pH monomer only associates into modest MW quaternary structures. Aggregation of proteins is generally assumed to be caused by the insolubility of the partially denatured state. Exposure of the hydrophobic core results in less-specific interactions, leading to modest MW aggregates, as is the case when TTR assembles over the range of $\mathrm{pH} 3$ to 2 in the presence of salt. In other cases aggregation has been shown to be highly specific, most likely resulting from the self-assembly of a structurally well-defined folding intermediate $(36,55,56)$, as in the case of TTR amyloid fibril formation at $\mathrm{pH} 4.4(26,29,32)$. The self-assembly of a conformational intermediate appears to yield amyloid fibrils and prion particles in a number of diseases $(1,6,26,57-$ $60)$. The results from this study, together with results from 
previous studies, $(1,23,24,26-29,32,61-63)$, suggest that the TTR amyloidogenic intermediate is a monomeric species having a defined but nonnative tertiary structure that can readily self-assemble into amyloid, likely through a ladder of quaternary structural intermediates.

\section{ACKNOWLEDGMENT}

We are grateful to W. F. Stafford for technical advice, T. O. Baldwin and the Center of Macromolecular Design at Texas A\&M for generous allocation of ultracentrifuge time, B. Demeler for technical advice and many helpful discussions regarding the van Holde-Weischet analysis, M. Scholtz for CD time, Jennifer Inlow for collecting some of the nearand far-UV CD data, Linda Woo for recording the EM images, and E. M. Wilson-Kubalek for EM advice.

\section{SUPPORTING INFORMATION AVAILABLE}

One figure showing the Congo red absorbance spectra in the absence and presence of L55P TTR (1 page). Ordering information is given on any current masthead page.

\section{REFERENCES}

1. Kelly, J. W. (1996) Curr. Opin. Struct. Biol. 6, 11-17.

2. Kelly, J. W. (1998) Curr. Opin. Struct. Biol. 8, 101-106.

3. Kelly, J. W. (1997) Structure 5, 595-600.

4. Lansbury, P. T. (1992) Biochemistry 31, 6865-6870.

5. Jacobson, D. R., and Buxbaum, J. N. (1991) Adv. Human Genet. 20, 69-123.

6. Booth, D. R., Sunde, M., Bellotti, V., Robinson, C. V., Hutchinson, W. L., Fraser, P. E., Hawkins, P. N., Dobson, C. M., Radford, S. E., Blake, C. C. F., and Pepys, M. B. (1997) Nature 385, 787-793.

7. Colon, W., Lai, Z., Lashuel, H. A., McCulloch, J., McCutchen, S. L., Miroy, G. J., Peterson, S. A., and Kelly, J. W. (1997) in Protein Misassembly (Wetzel, R., Ed.) pp 161-181, Academic Press, New York.

8. Sipe, J. D. (1992) Annu. Rev. Biochem. 61, 947-975.

9. Sipe, J. D. (1994) Crit. Rev. Clin. Lab. Sci. 31, 325-354.

10. Selkoe, D. J. (1997) Science 275, 630-631.

11. Golde, T. E., Estus, S., Younkin, L. H., Selkoe, D. J., and Younkin, S. G. (1992) Science 255, 728-730.

12. Shoji, M., Golde, T. E., Ghiso, J., Cheung, T. T., Estus, S., Shaffer, L. M., Cai, X.-D., McKay, D. M., Tintner, R., Frangione, B., and Younkin, S. G. (1992) Science 258, 126129.

13. Cohen, A. S., and Calkins, E. (1959) Nature 183, 1202-1203.

14. Cohen, A. S., Shirahama, T., and Skinner, M. (1982) in Electron Microscopy of Proteins (Harris, L. R., Ed.) pp 165205, Academic Press, London.

15. Shirahama, T., and Cohen, A. S. (1967) J. Cell Biol. 33, 679708 .

16. Stine, W. B., Snyder, S. W., Ladror, U. S., Wade, W. S., Miller, M. F., Perun, T. J., Holzman, T. F., and Krafft, G. A. (1996) J. Protein Chem. 15, 193-203.

17. Lambert, M. P., Barlow, A. K., Chromy, B. A., Edwards, C., Freed, R., Liosatos, M., Morgon, T. E., Rozovsky, I., Trommer, B., Viola, K. L., Wals, P., Zhang, C., Finch, C. E., and Krafft, G. A. (1998) Proc. Natl. Acad. Sci. U.S.A. 95, 6448-6453.

18. Harper, J. D., Wong, S. S., Lieber, C. M., and Lansbury, P. T. (1997) Chem. Biol. 4, 119-125.

19. Tan, S. Y., and Pepys, M. B. (1994) Histophathology 25, 403414.

20. Wetzel, R. (1991) in Stabilty of Protein Pharmaceuticals: In Vivo Pathways of Degradation and Strategies for Protein Stabilization (Ahern, T., and Manning, M., Eds.), Plenum, New York.

21. Westermark, P., Sletten, K., Johansson, B., and Cornwell, G. G. (1990) Proc. Natl. Acad. Sci. U.S.A. 87, 2843-2845.

22. Saraiva, M. J. M. (1995) Hum. Mutat. 5, 191-196.
23. McCutchen, S., Colon, W., and Kelly, J. W. (1993) Biochemistry 32, 12119-12127.

24. McCutchen, S. L., Lai, Z., Miroy, G., Kelly, J. W., and Colon, W. (1995) Biochemistry 34, 13527-13532.

25. Benson, M. D. (1989) TIBS 12, 88-92.

26. Colon, W., and Kelly, J. W. (1992) Biochemistry 31, 86548660.

27. Kelly, J. W., and Lansbury, P. T. J. (1994) Amyloid: Int. J. Exp. Clin. Invest. 1, 186-205.

28. Miroy, G. J., Lai, Z., Lashuel, H., Peterson, S. A., Strang, C., and Kelly, J. W. (1996) Proc. Natl. Acad. Sci. U.S.A. 93, $15051-15056$.

29. Lai, Z., Colon, W., and Kelly, J. W. (1996) Biochemistry 35, 6470-6482.

30. Lai, Z., McCulloch, J., and Kelly, J. W. (1997) Biochemistry 36, 10230-10239.

31. Inlow, J., Lai, Z., and Kelly, J. W. (1998) Biochemistry (submitted for publication).

32. Nettleton, E. J., Sunde, M., Lai, Z., Kelly, J. W., Dobson, C. M., and Robinson, C. V. (1998) J. Mol. Biol. 281, 553-564.

33. Serpell, L. C., Sunde, M., Fraser, P. E., Luther, P. K., Morris, E., Sangren, O., Lundgren, E., and Blake, C. C. F. (1995) J. Mol. Biol. 254, 113-118.

34. Blake, C., and Serpell, L. (1996) Structure 4, 989-998.

35. Quintas, A., Saraiva, M. J. M., and Brito, R. M. M. (1997) FEBS Lett. 418, 297-300.

36. Speed, M. A., Wang, D. C., and King, J. (1995) Protein Sci. 4, 900-908.

37. Snyder, S. W., Ladro, U. S., Wade, W. S., Wang, G. T., Barret, L. W., Matayoshi, E. D., Huffaker, H. J., Krafft, G. A., and Holzman, T. F. (1994) Biophys. J. 67, 1216-1228.

38. Lomakin, A., Chung, D. S., Benedek, G. B., Kirschner, D. A., and Teplow, D. B. (1996) Proc. Natl. Acad. Sci. U.S.A. 93, 1125-1129.

39. Shen, C. L., Fitzgerald, M. C., and Murphy, R. M. (1995) Biophys. J. 67, 1238-1246.

40. Shen, C.-L., and Murphy, R. M. (1995) Biophys. J. 69, 640651.

41. Stafford, W. F. (1994) Methods Enzymol. 240, 478-501.

42. van Holde, K. E., and Weischet, W. O. (1978) Biopolymers 17, 1387-1403.

43. Hansen, J. C., Lebowitz, J., and Demeler, B. (1994) Biochemistry 33, 13155-13163.

44. Laue, T. (1992) in Analytical Ultracentrifugation in Biochemistry and Polymer Science (Harding, S. E., Rowe, A. J., and Horton, J. C., Eds.) pp 63-89, Redwood Press Ltd., Cambridge, England.

45. Durchschlag, H. (1986) in Thermodynamic data for Biochemistry and Biotechnology (Hinz, H.-J., Ed.) Chapter 3, pp 45, Springer-Verlag, New York.

46. McMeekin, T. L., and Marshall, K. (1952) Science 116, 142145.

47. Perkins, S. J. (1986) Eur. J. Biochem. 157, 169.

48. Philo, J. S. (1994) in Modern Analytical Ultracentrifugation (Schuster, T. M., and Laue, T. M., Eds.) pp 156-170, Birkhauser, Boston.

49. Demeler, B., Saber, H., and Hansen, J. C. (1997) Biophys. J. 72, 397-407.

50. Stafford, W. F. (1992) Anal. Biochem. 203, 295-301.

51. Goto, Y., and Fink, A. L. (1989) Biochemistry 28, 945-952.

52. Goto, Y., Calciano, L. J., and Fink, A. L. (1990) Proc. Natl. Acad. Sci. U.S.A. 87, 573-577.

53. Goto, Y., Takahashi, N., and Fink, A. L. (1990) Biochemistry 29, 3480-88.

54. Fernando, T., and Royer, C. (1992) Biochemistry 31, 34293441 .

55. Goldberg, M. E., Rudolph, R., and Jaenicke, R. (1991) Biochemistry 30, 2790-2797.

56. King, J. (1986) Bio/Technology 4, 297-303.

57. Caughey, B., Kocisko, D. A., Raymond, G. J., and Lansbury, P. T. (1995) Chem. Biol. 2, 807-817.

58. Lansbury, P. T., and Caughey, B. (1996) Curr. Biol. 6, 914916. 
59. Patino, M. M., Liu, J.-J., Glover, J. R., and Lindquist, S. (1996) Science 273, 622-626.

60. Thomas, P. J., Qu, B. H., and Pederson, P. L. (1995) Trends Biochem. Sci. 20, 456-459.

61. Prusiner, S. B. (1997) Science 278, 245-251.
62. Baldwin, M. A., Cohen, F. E., and Prusiner, S. B. (1995) J. Biol. Chem. 270, 19197-19200.

63. Nguyen, J., Baldwin, M. A., Cohen, F. E., and Prusiner, S. B. (1995) Biochemistry 34, 4186-4192.

BI981876+ 